Aus der Nervenpoliklinik der Charité.

\title{
Über Hemiatrophie und Hemihypertrophie nebst einigen Bemerkungen über ihre laterale Lokalisation.
}

\author{
Von \\ Dr. Ewald Stier, \\ Stabsarzt and Privatdozent. \\ (Mit 10 Abbildungen.)
}

I.

Erna S., 11 Jahre alt, wurde uns von der Mutter am 5. Oktober 1910 in unserer Poliklinik vorgestellt wegen nervöser Beschwerden und Charakterstörungen, gleichzeitig aber auch wegen einer allmählich entstandenen Verkleinerung der rechten Gesichtshälfte. Sie wurde dann für 14 Tage, vor allem zur genaueren Beobachtung der angeblichen Charakterstörungen, in die Klinik aufgenommen und steht scitdem bis jetzt in poliklinischer Behandlung.

Aus der Vorgeschichte ist zu bemerken, dass die Eltern seit der Geburt des Kindes getrennt leben und später gerichtlich geschieden worden sind wegen einer geschlechtlichen Krankheit des Mannes. Es handelt sich dabei, wie mit ziemlicher Sicherheit festgestellt werden konnte, nicht um Lues, sondern um Gonorrhöe. Wie weit im nubrigen der Vater als nervös krank oder gesund anzusehen ist, entzieht sich unserer Kenntnis. Die Mutter soll eine hochgradig nervöse Frau sein, die nach Angabe des Mannes an nächtlichen Erregungszuständen mit Neigung zu impulsiven Handlungen leidet. Von den beiden etwa 8 und 9 Jahre älteren Schwestern des Kindes soll die ältere gesund sein, die jüngere, die wir selbst kennen, leidet an einer starken Struma mit viel Herzklopfen, Angstzuständen und Ohnmachtsanfällen.

Das Kind selbst ist rechtzeitig geboren; wegen Wehenschwäche der Mutter wurde die Zange angelegt und damit das Kind leicht und schnell geboren. Es war nicht asphyktisch. Die Nachgeburt war nicht angewachsen. Schnupfen oder Ausschlag wurden in den ersten Lebensmonaten nicht beobachtet. Der Schädel soll keinerlei Deformitäten gezeigt haben. Die erste Entwicklung erfolgte ohne Störung. Wegen mannigfacher Krankheit blieb das Kind später in der Entwicklung zurück, lërnte erst mit $1 \frac{1}{1} / 2$ Jahren laufen und mit $21 / 2$ Jahren sprechen. Die Sprache soll auch dann noch lange stammelnd gewesen sein. Das Kind bevorzugte von jeher und ausschliesslich die linke Hand. Auf der Schule lernte sie gut, blieb aber hinter den Altersgenossen wegen erneuter Krankheiten - angeblich Lungentuberkulose und Brustfellentzündung - zurück und sitzt jetzt erst in der 5. Klasse.

Seit 1908 wurde eine Veränderung des nervösen Verhaltens beobachtet. 
Der Schlaf wurde schlecht, sie schrak nachts auf, schimpfte, weinte und lief im Zimmer umher, war bei Tage reizbar, ungehorsam, unverträglich und zänkisch, besonders zu der Schwester. In der Schule benahm sie sich schliesslich so, dass ihre Wiederaufnabme nach der letzten Krankheit wegen ihres Betragens verweigert wurde. Zu Hause soll sie oft apathisch und stumpf, oft unmässig erregt gewesen sein, bald Suicid-Absichten geäussert haben, bald grosse Pläne für die Zukunft entwickelt haben. Dabei sollen Gedächtnis und Schulleistungen deutlich schlechter und sie sogar zur Erledigung kleiner Aufträge unfähig geworden sein.

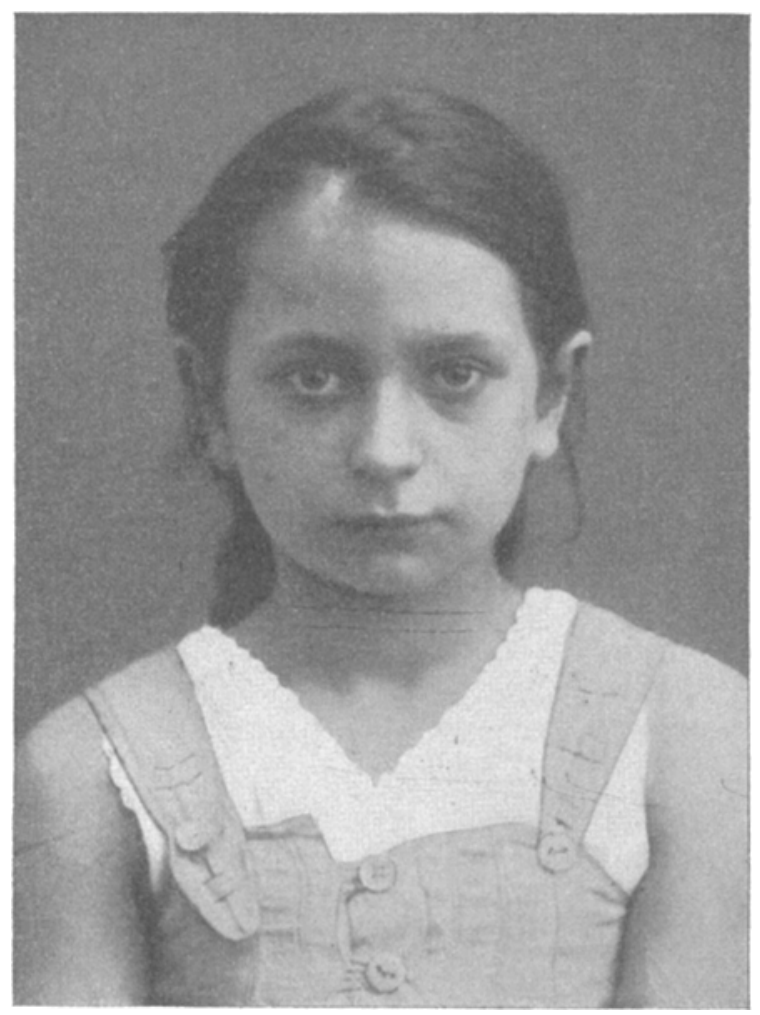

Fig.1.

Seit dem Hẻrbst 1909 wurde ein Einsinken der rechten Stirn und später der Wangengegend bemerkt, das von einer Stelle an der Haargrenze rechts dicht neben der Mittellinie seinen Ausgang nahm.

Befund (siehe Fig. 1): Schwächliches Kind ohne nachweisbare Krankheiten an den inneren Organen. Wassermann negativ. Die Pupillen sind gleich weit, die Reflexe sämtlich o. B. Sensibilität intakt, keine Störungen der Schweiss- oder Talgdrüsen-Sekretion. Intelligenz und Schulkenntnisse dem Alter entsprechend. In der ganzen rechten Stirnhälfte ist die Haut deutlich verdünnt, leicht pigmentiert, die Gefässe sind deutlich durch- 
scheinend, der Knochen darunter ist atrophisch. Die rechte Lidspalte ist etwas weiter als die linke. Knochen und Haut des Oberkiefers sind weniger, aber noch immer deutlich atrophisch, auch die rechte Nasenhälfte ist nicht ganz verschont. An der Stirn reicht die Grenze einige Millimeter über die Mittellinie hinüber. Weitaus am stärksten betroffen ist eine etwa $2 \mathrm{~cm}$ lange, $1 \mathrm{~cm}$ breite, oval geformte Stelle an der Haargrenze, an der die Haare gänzlich fehlen, die Haut ausserordentlich verdünnt und der Knochen so vertieft ist, dass man die Fingerkuppe in dieses Loch hineinlegen kann. Beim Abtasten der Umgebung dieser Stelle hat man das Gefühl, als ob der Knochen dort unregelmässig verdickt ist. Die Mutter gibt ausdrücklich an, dass diese am meisten betroffene Stelle niemals Sitz einer Wunde mit späterer Narbe gewesen ist. Beide Gesichtshälften fühlen sich gleich warm an. Tränenabsonderung r. $=1$.

In psychischer Beziehung ergab die Beobachtung lediglich die Zeichen einer psychopathischen Konstitution mässigen Grades. Das Benehmen in der Klinik war durchaus korrekt. Zeichen einer Geistesstörung im engeren Sinne des Wortes fanden sich nicht. -

Es handelt sich also um ein wahrscheinlich nicht hereditär luetisches, aber in nervöser Beziehung erblich belastetes 11 jähriges Mädchen, das sich spät entwickelt hat und viel körperlich krank gewesen ist und bei dem sich seit $1^{1 / 2}$ Jahren, gleichzeitig mit nerrösen allgemeinen Störungen und einer Charakterveränderung eine typische Hemiatrophia faciei entwickelt hat. Irgend eine Krankheit oder eine Läsion, die ursächlich in Betracht käme, ist dem Beginn der Atrophie nicht vorhergegangen. Schmerzen haben nie bestanden. Das erkrankte Gebiet überschreitet höchstens um Millimeter nach der Mitte zu das Innervationsgebiet des rechten Trigeminus. Die Erkrankung hat an scharf umschriebener Stelle an der Haargrenze begonnen und sich von dort allmählich, vor allem in der Richtung nach dem Gesicht zu, ausgebreitet. Atrophisch sind die Haut und noch mehr der darunter liegende Knochen; Zunge und Gaumen sind völlig frei. Die sensiblen oder sekretorischen Funktionen sind nicht beteiligt. Das Leiden ist bis jetzt dauernd progredient.

Die noch immer strittige Frage nach dem Sitz und der eigentlichen Ursache der Krankheit kann durch den vorliegenden Fall kaum gefördert werden. Unter keinen Umständen bildet er eine Bestätigung für die vor allem von Jendrassik ${ }^{1}$ ) angeregte und neuerdings von Oppenheim ${ }^{2}$ ) wenn auch nicht anerkannte, so doch infolge eines günstigen Operationserfolges wieder mehr in den Vordergrund des Interesses gerückte Frage nach dem Einfluss des Sympathicus auf die Entstehung der Krankheit. Denn es fehlen in unserem Falle alle

1) Jendrassik, Über die Hemiatrophia facialis. Archiv f. klin. Medizin. Bd. 59 .

2) Oppenheim, Lehrbuch, 5. Aufl. II. 1548. 
Zeichen für eine Beteiligung des Sympathicus, sowohl bezüglich seiner vasomotorischen als auch seiner sekretorischen Funktionen.

Die von Möbius ${ }^{1}$ ) vertretene Anschaung, dass eine rein exogene örtliche Schädigung infektiöser Natur die Krankheit hervorzurufen imstande sei, würde durch unseren Fall nicht widerlegt werden können, da die anfängliche Beschränkung und dauernd intensirste Beteiligung einer umschriebenen Hautstelle hier sehr deutlich zutage tritt. Doch ist es auch hier wie in allen bisher beobachteten Fällen nicht möglich, irgend eine Eingangspforte des supponierten Giftstoffes zu erkennen oder über dessen Natur auch nur Vermutungen anzustellen.

Die weitere, durch die Sektionsbefunde von $\mathrm{Mendel}^{2}$ ) und Homén ${ }^{3}$ ) etwas, durch den Sektionsbefund von Löbl und Wiesel ${ }^{4}$ ) erheblich mehr wahrscheinlich gemachte Annahme, dass der Trigeminus, speziell das Ganglion Gasseri als Sitz der Erkrankung anzusehen seien, würde klinisch insofern eine Bestätigung finden, als die Atrophie auch in unserem Fall sich fast genau auf das Gebiet des rechten Trigeminus beschränkt. Gegen den Versuch, durch eine Erkrankung des genannten Ganglion die Symptome der Krankheit zu erklären, spricht jedoch in unserem, wie in den meisten sonst beobachteten Fällen, das Fehlen aller sensiblen Reiz- oder Ausfallserscheinungen und wohl auch die Tatsache, dass $\mathrm{m}$. W. niemals bei den heutzutage so häufigen Operationen am peripheren Trigeminus oder dem Ganglion eine nachfolgende Hemiatrophie beobachtet worden ist. Doch muss zugegeben werden, dass durch die ganz verschiedene Intensität und Schnelligkeit der Einwirkung bei Operationen und bei den hier in Betracht kommenden Schädigungen der Jetztere Einwand etwas an Beweiskraft verliert. Dass eine Affektion des Trigeminus nicht die einzige und wesentliche Ursache der Krankheit sein kann, dürfte jedoch durch die mannigfachen Beobachtungen eines Hinausgehens des krankhaften Prozesses über das Gebiet dieses Nerven recht wahrscheinlich gemacht werden. Ich erinnere nur daran, dass u. a. Gowers in 2 Fällen Mitbeteiligung des Processus mastoideus und der Knochen an der Schädelbasis gefunden hat, dass in dem Falle von Leskowski das Gebiet der Halsnerven, von Heinemann die Brust-

1) Möbius, Der umschriebene Gesichtsschwund. Nothnagels spezielle Pathologie. XI. II. 2.

2) Mendel, Zur Lehre von der Hemiatrophia facialis. Neurolog. Zentralblatt 1888.

3) Homén, Zur Kenntnis der Hemiatrophia facialis und des Ursprungs des Nervus trigeminus. Neurolog. Zentralbl. 1890.

4) Löbl und Wiesel, Zur Klinik und Anatomie der Hemiatrophia facialis progressiva. Deutsche Zeitschr. f. Nervenheilkunde. Bd. 27. 1904. S. 355 . 
drüse, Williamson die Clavicula, Körner die nicht rom Trigeminus innervierten Teile der Ohrmuschel, Salomon die Augenmuskeln und Eulenburg die Sehnerven an der Erkrankung mitbeteiligt waren.

Als eine fast unerlässliche Voraussetzung für die Erklärung der Hemiatrophie durch eine Erkrankung des Trigeminus und seines Ganglion ist ferner die Existenz von trophischen Fasern im Verlauf dieses Nerven von den meisten Autoren angesehen worden, eine Annahme, für die besonders Samael mit Energie eingetreten ist. Es dürfte jedoch den eingehend begründeten Schlussfolgerungen von Cassirer ${ }^{1}$ ) beizutreten sein, dass trotz der bekannten vasomotorischen und trophischen Störungen bei krankhaften Durchtrennungen peripherer Nerven und trotz der experimentellen Ergebnisse von Lapinsky ${ }^{2}$ ) am Ischiadicus von Hunden und den Versuchen von Hautnervendurchtrennung, die $\mathrm{Head}^{3}$ ) bei sich selbst angestellt hat, die Existenz von besonderen, lediglich der Trophik dienenden Fasern innerhalb der peripheren Nerven nicht zur Erklärung der Erscheinung notwendig ist. Die genannten Erwägungen von Cassirer dürften auch für unseren Fall zutreffen, obwohl er bei dem Fehlen von jeglichen sensiblen Reiz- oder Ausfallerscheinungen im Gebiet des Trigeminus in besonderen Maße zur Annahme von der Existenz derartiger Fasern verführt.

Auch für die Berechtigung oder Nichtberechtigung der letzten von den wichtigeren Theorien über die Hemiatrophie, der zuerst von Brissaud aufgestellten, kann unser Fall uns nichts sagen. Die Brissaudsche Theorie, die von Calmette und Pagès und neuerdings anch von Kopczynsky angenommen worden ist, dass nämlich die Hemiatrophie als eine Syringoencephalie anzusehen sei und auf einer Affektion der periependymären grauen Substanz in der Oblongata und dem Pons beruhe, dürfte an sich zwar diskutabel sein, ihm fehlen aber anatomische Befunde noch ganz, und in den meisten bisher beobachteten Fällen einer Syringomyelobulbie, wohl mit alleiniger Ausuahme des Falles ron Schlesinger ${ }^{4}$ ), sind hemiatrophische

1) Cassirer, Die trophischen Störungen, im "Handbuch der Neurologie“, herausgegeben von Lewandowsky. Berlin 1910.

2) Lapinsky, Zur Frage über die Beteiligung der Nervenstämme der hinteren Extremitäten an der vasomotorischen Innervation der tieferen Gebiete derselben und über die Veränderung der vasomotorischen Elemente, sowie der Gefässe selbst der Hinterpfoten nach Beschädigung des Nervus ischiadicus. Virchows Archiv 183. 1906.

3) Rivers und Head, A human experiment in nerve division. Brain 1908. Nov.

4) Schlesinger, Über Spaltbildungen der Medulla oblongata und über 
Prozesse im Gesicht nicht beobachtet worden. - Auf die Berechtigung an eine noch höher, nämlich im Grosshirn, sitzende Störung zu denken, komme ich nachher zurïck. -

Nicht genügend gew ürdigt zu sein, scheint mir nun in der bis jetzt vorliegenden Literatur eine zwar schon öfter erwähnte, aber niemals zahlenmässig begründete Tatsache, nämlich die Regelmässigkeit, mit der in der weitaus überwiegenden Zahl der Fülle die gleiche Seite des Gesichts betroffen ist. Klar hingewiesen haben auf diese Bevorzugung der linken Gesichtshälfte u. a. Rotter, Lange, Beer, Eulenburg und Lewin.

Ein Versuch von mir, die Frage zahlenmässig zu verfolgen, hat nun zu folgendem Ergebnis geführt: von den 68 Fällen, die Lewin in den Charité-Annalen 1884 aus der Literatur zusammengestellt hat, hatten ihren Sitz, entsprechend den Angaben, die er bei den einzelnen Fällen macht, rechts 24 , links 44 . Von weiteren Fällen, die ich aus der späteren Literatur selbst gesammelt und in eine Tabelle eingetragen habe, hatten ihren Sitz rechts 18, links 40.

Diese meine Zusammenstellung macht natürlich nicht den Anspruch, vollständig zu sein und sämtliche in der Literatur niedergelegten Fälle ausnahmslos zu enthalten, sie ist jedoch vollständig insofern als alle Fälle, die ich in der Literatur aufgefunden habe, auch in diese Tabelle eingetragen sind. Um eine Kontrolle zu ermöglichen, habe ich unten ${ }^{1}$ ) die Namen der in betracht kommenden Autoren angegeben.

Mit hinzurechnen zu diesen Fällen möchte ich weiterhin die von Eulenburg beobachteten 10 Fälle, über die er in der letzten Auflage seiner Realenzyklopädie berichtet und in denen nur einmal die Er-

die anatomischen Bulbärsymptome bei Syringomyelie. 1906 zit. nach Löbl und Wiesel. Siehe dazu auch den neuesten Fall von Donath in der Zeitschrift für Nervenheilkunde. 1910. Bd. 40. Heft 5/6.

1) Der Sitz der Krankheit betraf die linke Seite in folgenden Fällen: Aus den Jahren 1882: Riegel, Küster; 1886: Penzoldt; 1888: Mendel; 1890: Homén, Schweninger; 1891: Jolly; 1892: Karewsky; 1893: von Ziegenweidt; 1894: Denig, Baerwaldt, Zappert, Joseph; 1896: Schlesinger; 1897: Le Maire, Lunz; 1898: Hoeflmayer; 1900: Mourat off; 1901: Luxenburger, Jadassohn, Hoffmann; 1902: Koerner, Bouveyron (2 Fälle); 1903: Raymond et Sicard, Calmette et Pagès, Rutten, Debray; 1904: Parry, Löbl und Wiesel, Werner; 1905: Collins; 1906: Gowers (2 Fälle), Leskowsky; 1907: Heinemann, Salomon; 1908: Orbison, Williamson, Klieneberger. Der Sitz der Krankheit betraf die reehte Seite bei: 1886: Penzoldt; 1887: Martin; 1889: Cahen, Ephraim; 1891: Jankau; 1896: Decsi; 1897: Bruns; 1901: Hoffmann, Luxenburger; 1903: Fischer, Kopczynski; 1904: Hrach; 1906: Gowers, Wirschulski, Leskowsky, Herrenberg; 1907: Klingmann, Kopczynski. 
krankung ihren Sitz rechts, 9 mal dagegen nur links oder $1>\mathrm{r}$ hatte. Hinzurechnen möchte ich weiterhin die 4 Abbildungen in Oppenheims Lehrbuch, bei denen 3 mal die rechte und 1 mal die linke Seite erkrankt ist. Bei einer Zusammenstellung aller dieser Fälle ergibt sich folgende Tabelle.

\section{Tabelle 1.}

Lewin

Eulenburg

Oppenheim

Literatur

eigene Beobachtungen

\section{rechts links}

\begin{tabular}{rr}
24 & 44 \\
1 & 9 \\
3 & 1 \\
18 & 40 \\
1 & - \\
\hline 47 & 94
\end{tabular}

Es zeigt sich also, dass die Häufigkeit, mit der die linke oder rechte Seite betroffen ist, sich fast genau wie $2: 1$ verhält, d. h. dass die linke Gesichtshälfte doppelt so häufig von der Hemiatrophie betroffen wird als die rechte.

II.

L. B., 12 Jahre, kommt mit seiner Stiefmutter aus hanada, um hier wegen seines Leidens Rat und Hilfe zu finden. Die Stiefmutter, die ihn seit 5 Jahren kennt, gibt an, dass der Vater ein 45jähriger gesunder Mann ist, der aus ganz gesunder Familie stammt, dass die Mutter an Lungentuberkulose verstorben ist und zwei ältere Geschwister des Knaben und ein kleiner Stiefbruder gesund sind, im besonderen sollen Nerven- und Geisteskrankheiten oder Missbildungen irgend welcher Art niemals in der ganzen Familie beobachtet worden sein.

Die Geburt des Knaben ist rechtzeitig, glatt und ohne Kunsthilfe von statten gegangen. Gleich nach der Geburt schon fiel auf, dass das linke Auge zu gross war, es wurde deshalb, als der Knabe 1/2 Jahr alt war, eine Iridektomie vorgenommen ${ }^{1}$ ) und als das Ange weiterhin wuchs, im Jahre 1902 eine Enukleation gemacht.

Das Laufen erlernte Patient erst mit 3 Jahren, zu sprechen begann er gleichfalls mit 3 Jahren und beherrschte auch mit 6 Jahren die Sprache erst unvollständig. An protrahiertem Bettnässen oder nächtlichem Aufschrecken litt er nicht. In der geistigen Entwicklung blieb er dauernd zurück, lernte ziemlich schlecht und ungleichmässig und blieb, obwohl er erst mit 8 Jahren den Schulunterricht begann, wiederholt sitzen. Von Charakter soll er im allgemeinen gutartig sein, doch hat er von jeher eine etwas gesteigerte Neigung zum Lügen, hat auch einmal der Mutter Geld fortgenommen, dafür Schokolade gekauft, diese dann aber zum Teil verschenkt. Zu Tieren ist er meistens freundlich und gut, zuweilen jedoch

1) Mitteilung des damals behandelnden Arztes Dr. Glen Campbell in Vancouver. 
soll er auch Hunde und Katzen schlagen und treten. Er soll manchmal aufbrausend, im übrigen aber freundlich und liebevoll sein gegen Eltern und Geschwister. Masturbation oder psycho-sexuelle Frühreife ist nicht beobachtet worden. Von ernsteren Krankheiten hat er nur Keuchhusten und Masern durchgemacht. Kopfschmerzen, Erbrechen, Sehstörungen haben niemals bestanden.

Die jetzige Krankheit trat erst klar zutage nach der Entfernung des linken Auges im Jahre 1902. Sie bestand darin, dass das linke Augenlid, die Wange und Kiefergegend der linken Seite stärker wuchsen als die entsprechenden Teile der rechten Gesichtshälfte. Dieses vermehrte Wachstum war anfangs gering, in den letzten Jahren aber wurde es immer stärker, so dass das ganze Gesicht verunstaltet wurde; auch zeigte sich an der linken Wange und Oberlippe im Gegensatz zu dem sonst durchaus kindlichen Habitus des Knaben seit etwa einem Jahr Bartwuchs. Das

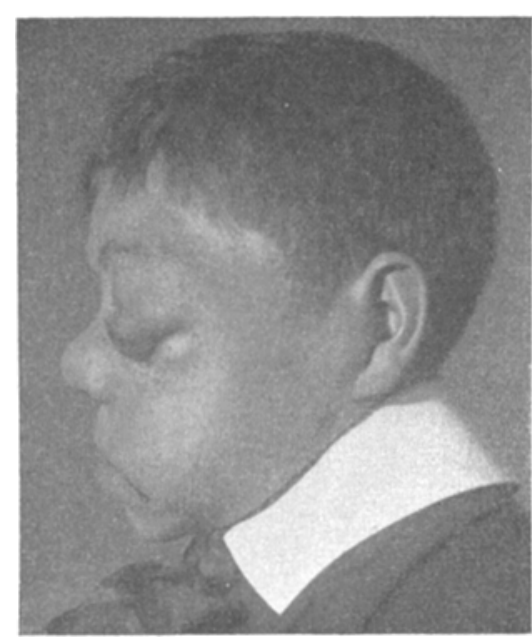

Fig. $2 \mathrm{a}$

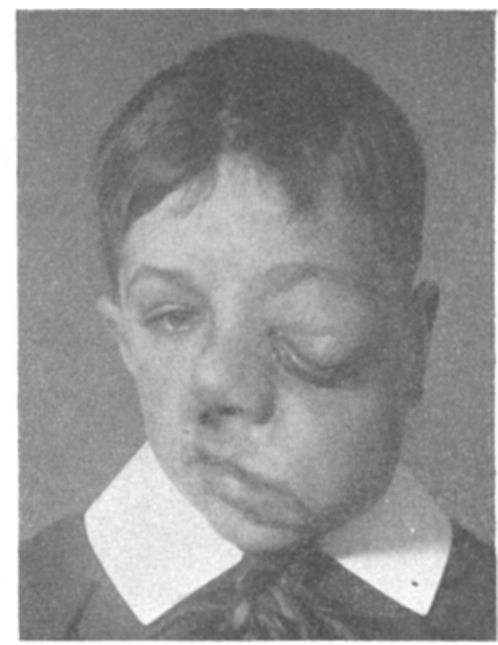

Fig. $2 b$.

Wachstum erstreckte sich auch auf die knöchernen Teile. Schmerzen bestanden spontan niemals, auch sonst keinerlei Beschwerden.

Befund (siehe Fig. 2) 28. März 1911: Mittelgrosser, seinem Alter entsprechend entwickelter Knabe. Die unteren Extremitäten sind gleich gross und normal gebildet, ihre Sehnenreflexe mittelstark und gleich. An der Haut des Rumpfes und der Oberschenkel finden sich mehrere linsenbis talergrosse Pigmentflecke von graubrauner Farbe. An den inneren Organen sind Grössenabweichungen, Verlagerungen oder sonstige krankhafte Erscheinungen nicht nachweisbar. Der 4. Finger der linken Hand ist vielleicht ein wenig länger als der der rechten Hand, auch ist die Grössendifferenz zwischen dem 2. und 3. Finger der linken Hand vielleicht etwas beträchtlicher als rechts. Im übrigen finden sich keine Unterschiede der oberen Extremitäten der rechten und der linken Seite. Der Knabe ist rechtshändig.

Die rechte Schädel- und Gesichtshälfte zeigt keinerlei Abweichungen 
von der Norm. Die Pupille ist rund; ihre Lichtreaktion prompt und ausgiebig.

Links ist der Schädel in seinen hinteren Teilen normal gebildet. Das Stirnbein dagegen ist in seinen oberen Teilen etwas, in der Gegend des oberen Augenhöhlenrandes erheblich verdickt, desgleichen zeigen der Arcus zygomaticus sowie der Ober- und der Unterkiefer sehr beträchtliche Vergrösserungen und Verdickungen. Man fühlt dabei Rauhigkeiten und Auswüchse des Knochens, die auf Druck schmerzhaft sind. Am stärksten tritt diese Verdickung am Processus alveolaris des Oberkiefers hervor, der ganz ausserordentlich verdickt und mehr als doppelt so breit ist als auf der rechten Seite. Trotzdem ist nur ein einziger Backzahn hier durchgebrochen und auch dieser ist nur von geringer Grösse. Auch im Unterkiefer finden sich im Gegensatz zur rechten Seite links nur ein Backzahn, obwohl nach Angabe der Mutter hier nirgends Zähne entfernt worden sind. Der Schädelumfang beträgt in der Riegerschen Ebene $52,5 \mathrm{~cm}$, der Längenbreitenindex $17,5: 13=74,2$.

Die Weichteile sind gleichfalls in der ganzen linken Gesichtsseite erheblich vergrössert.

Die Länge des Ohres beträgt $6 \mathrm{~cm}$ links gegen $5,5 \mathrm{~cm}$ rechts; die Gegend des Tragus ist besonders verdickt und vergrössert.

Das untere Augenlid ist etwas, das obere sehr stark vergrössert und ektropioniert; es hängt fast bis zur Höhe der unteren Nasengrenze herab. Bewegungen dieses Augenlides sind möglich, beschränken sich aber vorwiegend auf Zuckungen, die auch unwillkürlich auftreten; eine wirkliche Hebung des Lides ist nicht möglich. Das Auge selbst fehlt.

Die Schwellung der Haut in der linken Gesichtshälfte ist so stark, dass das ganze Gesicht verunstaltet, die Nase und die Mundgegend nach rechts weit verschoben sind. Dabei ist die Haut in ihrer Färbung kaum verändert; nirgends ist eine Geschwulst abgrenzbar, alles vielmehr teigig geschwollen, fühlt sich aber doch deutlich fester und derber an als bei ödematöser Schwellung; Fingereindrücke bleiben dementsprechend auch nicht stehen.

Abnorme Pigmentierungen der Haut des Gesichts sind nicht nachweisbar. Die Funktion der Talgdrüsen zeigt keine Veränderungen. Die Temperatur der Gesichtshaut ist auf beiden Seiten gleich. Nach Eingaben eines schweisstreibenden Mittels tritt auf der rechten Gesichtshälfte starke Schweisssekretion, auf der linken nur eine Erhöhung der Temperatur ein. Bei elektrischer Reizung des Ausführungsganges der Unterzungen-Speicheldrüse zeigt sich nur rechts Speichelabsonderung.

Der Haarwuchs ist deutlich vermehrt. Es findet sich sowohl in der Wangengegend unterhalb des Ohres, als auch auf der Oberlippe ein echter Bartwuchs, der scharf in der Mittellinie abschneidet; die Dicke der einzelnen Haare ist mikrometrisch gemessen $\mathbf{r}=1$.

Die Berührungs- und Schmerzempfindlichkeit ist im ganzen Gebiet des linken Trigeminus herabgesetzt gegen rechts. In den tubrigen Teilen des Schädels besteht vielleicht links eine etwas geringere Empfindlichkeit als rechts. Das Gleiche schien an den unteren Extremitäten der Fall zu sein, doch waren die Angaben hier sehr unsicher und kaum verwertbar. Die Untersuchung der Gesichtsmuskeln mit dem galvanischen Strom ergab 
eine geringe quantitative Herabsetzung der Erregbarkeit im Triangularis menti und Orbicularis oculi.

In psychischer Beziehung zeigt sich ein nicht erheblicher, aber deutlicher Intelligenzdefekt. Das Benehmen war im allgemeinen korrekt, doch bestand eine auffallende Neigung unmotiviert za lachen. Im engeren Sinne des Wortes psychotische Symptome fanden sich nicht.

Die anatomische Untersuchung der herausgeschnittenen Hautteile (Dr. Löhe) ergab eine Abflachung und fast völlige Atrophie der Papillen,

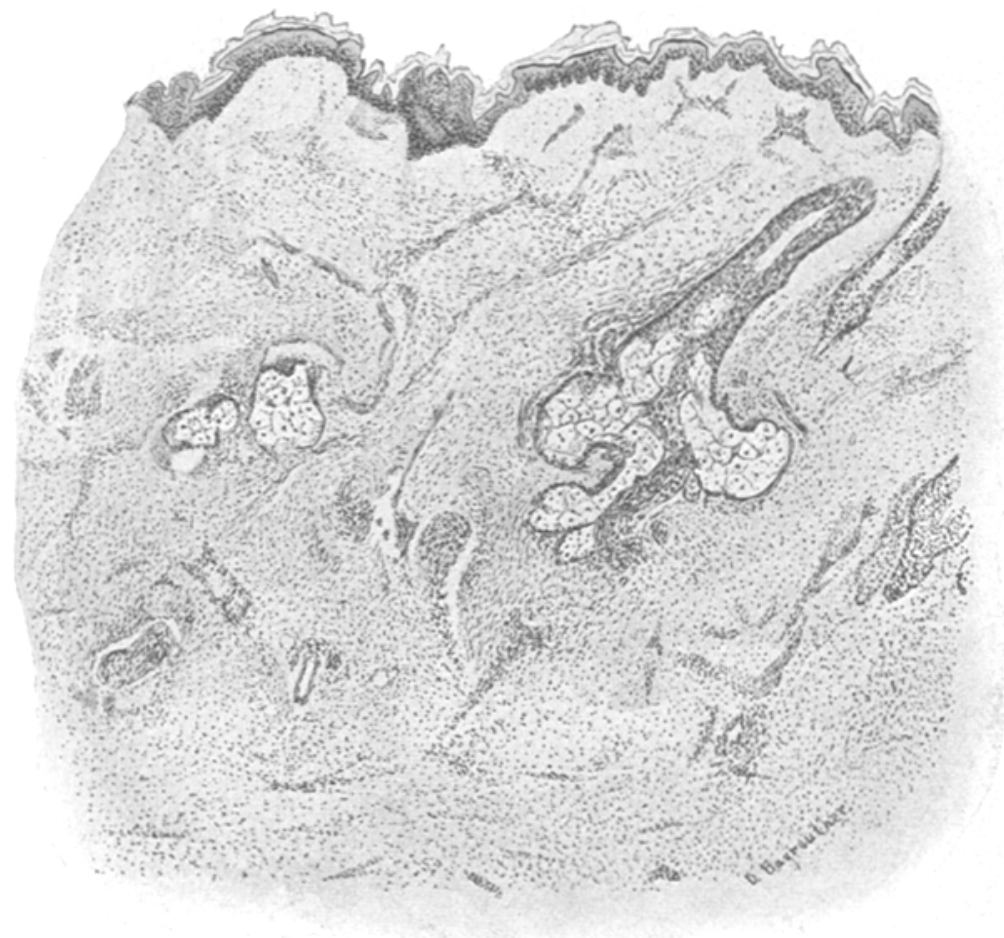

Fig. 2 e.

sowie eine Infiltration aller Teile der Haut bis in das Unterhautfettgewebe hinein mit kleinen, teils runden, teils spindeligen Bindegewebszellen, also ein der Elefantiasis durchaus entsprechendes Bild (siehe Figur 2c).

Zusammengefasst handelt es sich also um einen Knaben, bei dem, so weit wir haben feststellen können, ausser der Tuberkulose der Mutter schädigende erbliche Einflüsse nicht eingewirkt haben und dessen Geburt normal verlaufen sein soll. Schon gleich bei der Geburt bestand eine Vergrösserung des linken Auges, die bald zunahm und zur Entfernung des Auges führte. In den weiteren Jahren trat 
dann ohne weitere äussere Ursachen ein vermehrtes Wachstum der ganzen Knochen- und Weichteile der linken Gesichtshälfte ein, die zu völliger Verunstaltung des Gesichtes führte. Am meisten beteiligt sind von Knochen die Kiefer, der Jochbogen, etwas weniger das Stirn- und Schläfenbein. Der Zahnw uchs ist in der kranken Seite trotz erheblicher Hypertrophie der Alveolarfortsätze verringert. Die Weichteile sind teigig geschwollen, derber und fester als bei Ödem, aber doch weniger hart als bei dem Trophödem im Sinne von Meige. Die Sekretion der Speichel- und Schweissdrüsen ist verringert; die Schmerzhaftigkeit ist in den hypertrophischen Gebieten bestimmt, in den übrigen Teilen der linken Körperhälfte vielleicht herabgesetzt; die elektrische Erregbarkeit für den galvanischen Strom ist in den Muskeln der linken Gesichtshälfte quantitativ etwas geschädigt. An der linken Wange and Oberlippe findet sich im Gegensatz zu der rechten Seite und zu dem Alter des Knaben schon deutlicher Bartwuchs; die Dicke der einzelnen Haare ist dabei links nicht grösser als rechts.

In psychischer Beziehung hat sich Pat. spät und langsam entwickelt und ist noch jetzt nicht auf der Höhe der Altersgenossen. -

$\mathrm{Zu}$ der gleichen Zeit, als dieser Knabe zum Zweck einer kosmetischen Operation in die chirurgische Klinik verlegt worden war, wurde dort ein zweiter Fall der gleichen Krankheit aufgenommen, dessen Mitbenutzung mir von dem Direktor der Klinik, Herrn Geheimrat Professor Dr. Bier, gütigst gestattet wurde.

C. T., geboren 1906 , als Sohn eines Lehrers in Pommern. Soweit zu erfahren war, sollen Nervell- oder Geisteskrankheiten oder Missbildungen in der Familie nicht vorgekommen sein. Nach schriftlicher Mitteilung des Vaters ist das Kind rechtzeitig geboren, die Geburt selbst dauerte sechs Stunden und verlief ohne jede Störung und ohne ärztliche Hilfe; das Kind war nicht asphyktisch und schrie sofort. Schon gleich nach der Geburt fiel den Eltern auf, dass das rechte Auge grösser war als das linke. Als der Knabe $1 \frac{1 / 2}{2}$ Jahr alt war, begann der rechte Mundwinkel herabzụhängen, die rechte Wange schwoll an und das Auge wurde so gross, dass es in der hiesigen Augenklinik enukleiert wurde. In den folgenden Monaten und Jahren trat dann eine Schwellung und vermehrtes Wachstum der ganzen rechten Gesichtshälfte hinzu, bis der Vater jetzt erneut ärztliche Hilfe in der hiesigen Klinik suchte.

Im Sommer 1910 machte Patient ausserdem eine Mittelohreiterung auf dem rechten Ohr durch.

Befund (siehe Fig. 3) 10. Mai 1911: Dem Alter entsprechend entwickelter Knabe. An den unteren Extremitäten keinerlei Besonderheiten. Sehnenreflexe an Kinn und Ferse mittelstark, gleich. An der Haut der Brust finden sich mehrere bräunliche Pigmentflecke von rundlicher unregelmässiger Gestalt und einem Durchmesser von etwa $1-3 \mathrm{~cm}$. An den 
oberen Extremitäten finden sich keine Unterschiede zwischen rechts und links. Der Knabe ist ausgesprochen rechtshändig.

Schädel und Gesicht zeigen in der linken Hälfte keinerlei Abweichungen. Der Schädelumfang beträgt in der Riegerschen Ebene $521 / 2 \mathrm{~cm}$. Rechts ist der Schädel in der Gegend des Schläfenbeines deutlich stärker entwickelt als links. Die Augenhöhle erscheint erweitert, das Auge fehlt. Der obere Augenhöhlenrand zeigt besonders an seinem lateralen Ende spitze Knochenauswüchse mit tiefen Einbuchtungen dazwischen, in die man fast den Finger hineinlegen kann. Eine genaue Abgrenzung dieser Knochenveränderungen ist durch Palpation nicht möglich, da die darüber liegenden Weichteile gleichfalls verdickt sind und jeder Druck auf die Exostosen als

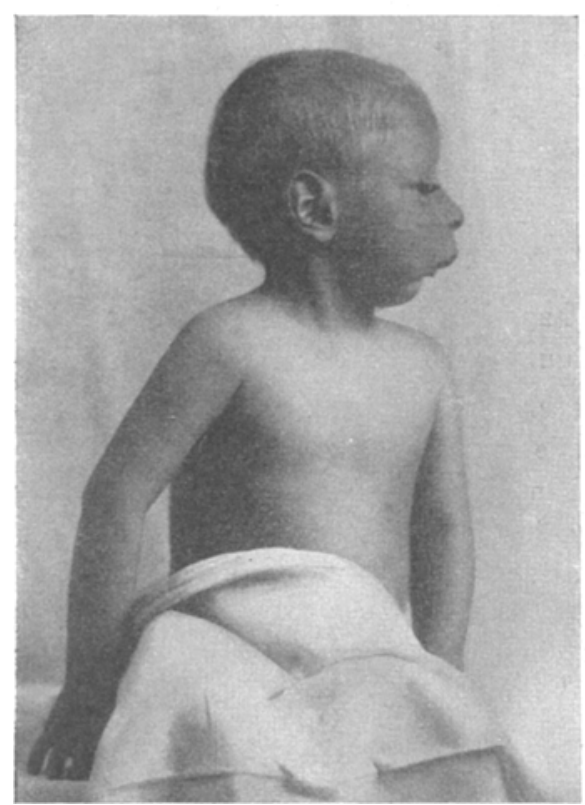

Fig. 3a.

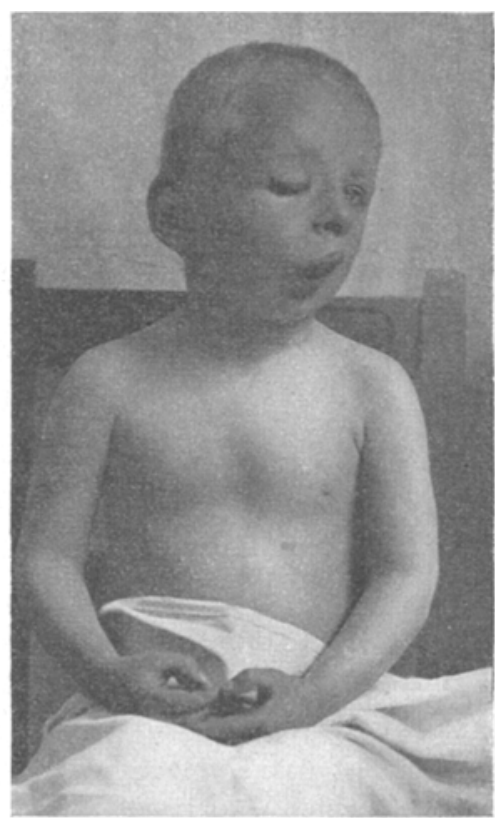

Fig. $3 b$

schmerzhaft empfunden und mit starken Abwehrreaktionen begleitet wird. Der Jochbogen ist in seiner ganzen Grösse verbreitert und verdickt. Der Oberkiefer ist in allen Teilen etwas, in seinem Alveolarfortsatz extrem vergrössert und verbreitert; seine Breite beträgt hier ungefähr $2 \frac{1}{1} \mathrm{~cm}$. Die Zahl der Backzähne ist auf beiden Seiten gleich, doch sind die einzelnen Zähne auf der rechten Seite erheblich kleiner als links. Der Unterkiefer zeigt nur eine geringe Verdickung.

Die Weichteile nehmen in erheblichem Maße an der Vergrösserung teil. Das Gesicht ist im ganzen nach links verschoben, die Mittellinie weicht besonders von der Gegend der Nasenspitze bis zum Kinn in bedeutendem Maße schräg nach links ab. Am meisten vergrössert und in toto verdickt ist die Gegend der Oberlippe und Wange nach dem Mundwinkel zu, etwas weniger Unterlippe und Schläfengegend, noch weniger 
die Stirn. Das obere Augenlid ist stark vergrössert und hängt weit über das untere, kaum vergrösserte Lid herab, so dass man das Fehlen des Bulbus zuerst kaum gewahrt., Die Haut ist in den genannten Gegenden überall von normaler Farbe, teigig geschwollen, fester als beim gewöhnlichen Ödem,

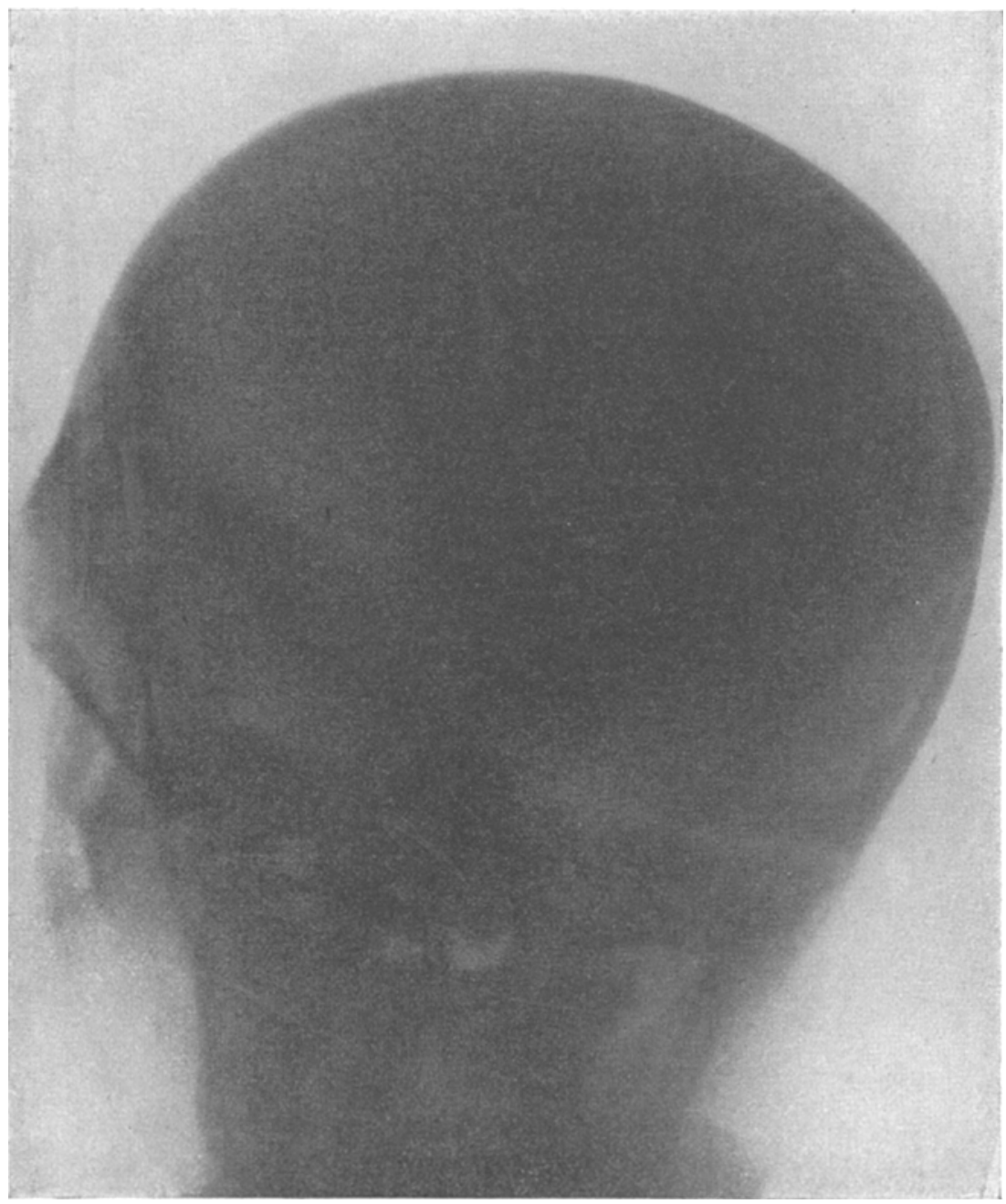

Fig. 3c.

aber in keiner Weise starr. Die Schleimhaut des Mundes an der Wange und dem Kieferfortsatz ist gleichfalls verdickt und trägt dazu bei, den Eindruck der massigen Vergrösserung noch zu erhöhen.

Die Zunge ist von durchschnittlicher Grösse und wird fast gerade Deutsche Zeitschrift 1 . Nervenheilkunde. Bd. 44. 
herausgestreckt. Die Länge der Ohren beträgt links 5,7 , rechts $6,0 \mathrm{~cm}$. Der Haarwuchs ist $r=1$.

Die Beruhhrungsempfindlichkeit ist nicht sicher zu prüfen. Die Schmerzempfindlichkeit scheint nicht wesentlich gestört zu sein. Es erfolgte überall schon auf leichte Stiche erhebliche Abwehrreaktion und Weinen, so dass eine genaue und eingehende Untersuchung auch bei wiederholten Proben nicht gelang. Eine Differenz der Schweiss- oder Speichelsekretion wurde nicht beobachtet, experimentelle Untersuchungen nach dieser Richtung konnten jedoch nicht vorgenommen werden. Eine Prüfung der elektrischen Erregbarkeit und weitere Spezialuntersuchungen unterblieben gleichfalls aus äusseren Gründen.

Gehör, Geruch, Gesicht sind, soweit zu prüfen, völlig intakt.

Die Sprache ist noch sehr unentwickelt. "f" wird meist ersetzt durch , $\mathrm{p}^{\text {" oder " }} \mathrm{h}$ ", "k" durch „t". Konsonantenverbindungen gelingen fast niemals. Patient sagt gün statt grün, bau oder brau statt blau. Sätze werden überhaupt nicht gebildet.

Die Bezeichnung von Gegenständen gelingt entsprechend der Sprachstörung nur ungenügend, doch werden die gewöhnlichen Gegenstände des Alltags offenbar richtig erkannt und in jedem Falle richtig benutzt; auch aus dem Bilderbuch werden einige besonders leicht erkennbare Bilder, wie Ball, Kamm, Eimer richtig erkannt, die übrigen, ein wenig schwereren jedoch falsch oder gar nicht bezeichnet. Geld wird als solches erkannt und freudig begrüsst mit den Worten: "Taufmann, (Schoko) lade taufen." Der Arzt wird als solcher leidlich verständlich bezeichnet.

Zum selbständigen Essen ist er im allgemeinen nicht fähig. Butterbrote und andere feste Speisen werden, wenn auch in wenig sauberer Weise, selbst gegessen, Suppe essen mit dem Löffel gelingt jedoch nicht; Patient wird daher fast bei allen Speisen gefüttert. Beim Spielen stellt er sich nicht ganz so ungeschickt an, ist jedoch völlig unfähig, in einer seinem Lebensalter entsprechenden Weise sich zu beschäftigen.

Zusammengefasst handelt es sich also um einen. 5 jährigen Knaben, der, soweit festzustellen war, nicht erblich belastet ist und der weder bei der Geburt noch nach der Geburt nennenswerte Schädigungen erfahren hat. Schon gleich nach der Geburt wurde eine Vergrösserung des rechten Auges bemerkt, die dauernd zunahm und im Alter von 11/4 Jahren die Entfernung des Auges nötig machte. Schon vorher und seitdem fortschreitend, zeigte sich eine Vergrösserung der rechten Kopf-und Gesichtshälfte, die am stärksten die Knochen des Jochbogens und der benachbarten Teile betraf und dort zu erheblichen Knochenauswüchsen führte, aber auch auf den Oberkiefer, den Unterkiefer und die Stirn- und Schläfengegend sich erstreckte, wenn auch in geringerem Maße. Die Weichteile zeigen in gleicher Weise eine erhebliche Vergrösserung in den genannten Gegenden, doch liegt die Gegend der stärksten Vergrösserung hier etwas tiefer, in der unteren Wangen- und Oberlippengegend. Die Haut hat dabei ihre normale Farbe und Spannung. 
behalten. Störungen in der Berührungs- und Sehmerzempfindlichkeit, in der Speichel- und Schweissabsonderung wurden nicht beobachtet. Die Zähne sind in der betroffenen rechten Kieferhälfte kleiner als links.

Die Sprache und die Intelligenz sind in ihrer Entwicklung zurückgeblieben. Betroffen ist ausschliesslich die rechte Gesichtshälfte bei dem rechtshändigen Kinde. -

Der dritte, in kurzer Frist in der Nerven-Poliklinik zugegangene Fall von Hemihypertrophie ist der folgende:

Erich R., $4 \frac{1}{2}$ Monate alt, in die Poliklinik aufgenommen am 1. Juni 1911. Nach der Angabe der Mutter sind weder bei ihr, noch bei ihrem Manne, noch in ihrer Verwandtschaft jemals Nerven- oder Geisteskrankheiten oder körperliche Missbildungen vorgekommen; auch Tuberkulose oder Alkoholismus soll niemals vorgekommen sein. Die Mutter hat vorher einmal, vor 2 Jahren, und zwar Zwillinge geboren, die gleich nach der Geburt gestorben sind. Sie waren klein und schwach, wohl etwas zu früh geboren, aber durchaus normal gebildet.

Die Geburt unseres Patienten trat rechtzeitig ein, sie verlief glatt und schnell im Laufe von 2 Stunden ohne ärztliche Hilfe. Das Kind, das in Schädellage geboren wurde, war „ziemlich dick“. Eine genauere Besichtigung liess sofort erkennen, dass die rechte Zungenhälfte sehr viel grösser war als die linke. Weitere Störungen wurden zunächst nicht bemerkt. Das Kind gedieh von Anfang an gut.

In den ersten Wochen fiel dann der Mutter auf, dass die rechte Wange stärker war als die linke, im Alter von 3 Monaten, dass das rechte Bein, und im 4. Monat, dass auch der rechte Arm stärker war als der linke. Die Differenz zwischen der rechten und linken Körperhälfte vergrösserte sich zunehmend, während die Differenz der Zungenhälfte keine Veränderung zeigte.

In der Farbe und sonstigen Beschaffenheit der Haut, sowie beim Schwitzen wurden halbseitige Unterschiede nicht bemerkt. Im ganzen entwickelte sich das Kind schnell und gut. Vater, Mutter, die Eltern und 4 Geschwister der Mutter sollen bestimmt rechtshändig sein, bei dem Kindchen selbst wurde, obwohl es schon anfängt, Gegenstände festzuhalten und auch danach zu greifen, eine Bevorzugung einer Hand bisher nicht bemerkt.

Befund (siehe Fig. 4): Kräftiges, dem Lebensalter mindestens entsprechend entwickeltes Kind. Schädelumfang $41 \mathrm{~cm}$. Länge etwa $63 \mathrm{~cm}$. Herzdämpfung in normalen Grenzen, Töne rein; an den Lungen oder Bauchorganen nichts Krankhaftes nachweisbar. Die Augen sind gleich gross. Pupillen mittelweit, gleich, Lichtreaktion prompt und ausgiebig. Augenbewegungen ruhig, frei. Die Sehnenreflexe sind an Ellenbogen, Kinn und Ferse mittelstark und gleich. Fusssohlenreflexe meist dorsalwärts. Auf Hautreize entsprechende Abwehrbewegungen.

Die Farbe und fühlbare Temperatur der Haut ist überall $r .=1$. Gefässerweiterungen oder Hautpigmentierungen finden sich nirgends. Keine Anomalie des Haarwuchses. Genitalien o. B. 
Das Kind nimmt an der Umgebung lebhaften Anteil, fixiert vorgehaltene Gegenstände, greift danach, hält sie fest; der Kopf wird zeitweise selbst aufrecht gehalten. Sensibilität, soweit feststellbar, intakt.

Schon beim Betrachten fällt auf, dass das rechte Bein und der rechte Arm länger und dicker sind als auf der linken Seite und dass auch der Rumpf und das Gesicht rechts etwas stärker ist als links.

Im einzelnen ergibt die Untersuchung, dass die rechte Wangen-

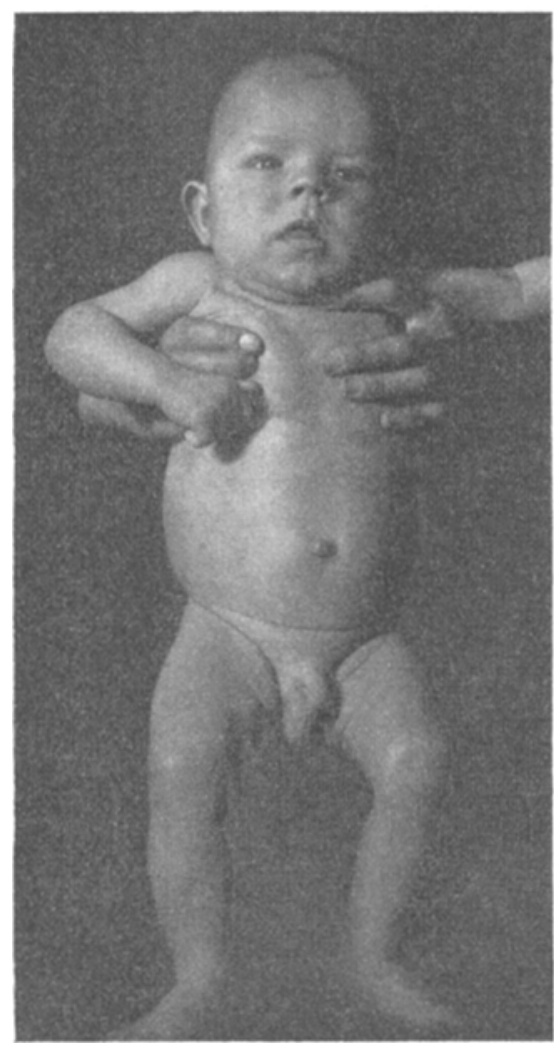

Fig. $4 \mathrm{a}$.

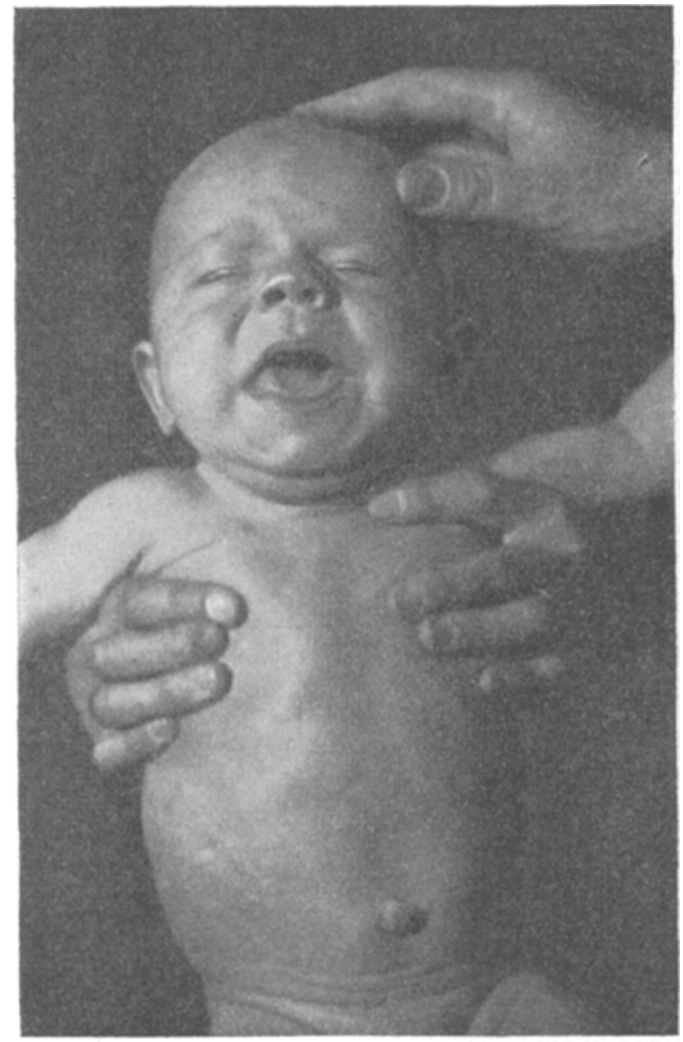

Fig. 4 b.

gegend, der Jochbogen und der untere Augenhöhlenrand mit den darüber liegenden Weichteilen etwas stärker entwickelt sind als links.

Die Zunge zeigt eine völlige Verschiebung der Mittellinie bis nach der Gegend des linken Mundwinkels. Die linke "Hälfte" scheint dabei von normaler Grösse zu sein, während die rechte ganz ausserordentlich verbreitert und ausserordentlich verdickt ist (siehe Abbildung). Beim Schreien tritt diese Differenz in vollem Umfange zutage, besonders durch die starke Einziehung der Mittellinie dabei.

Die Ohrmuscheln weisen keine messbare Differenz auf. Die Gaumen- 
bögen erscheinen gleich gross. Die Kiefer zeigen keine sichere Differenz. Zähne fehlen noch.

Der $R$ u mpf zeigt von der Mitte des Brustbeins bis zu dem Dornfortsatze auf gleieher Höhe, rechts wie links gemessen, folgende Umfänge: dicht oberhalb der Brustwarzenlinie $\mathrm{r}=21,5, \mathrm{l}=2020,0 \mathrm{~cm}$; in der Mitte 2wischen Nabel und Schwertfortsatz $\mathrm{r}=22,5, \mathrm{l}=21,5 \mathrm{~cm}$.

Das Bein, gemessen von der Spina ant. sup. bis zum äusseren Fussknöchel: eine Länge von $\mathrm{r}=26, \mathrm{l}=25 \mathrm{~cm}$; grösster Umfang der Wade $\mathrm{r}=15, \mathrm{l}=13 \mathrm{~cm}$; Umfang des Oberschenkels $5 \mathrm{~cm}$ oberhalb der Kniescheibe $\mathrm{r}=20^{1 / 4}, \mathrm{l}=18 \mathrm{~cm}$.

Der Arm, gemessen von der Achselhöhle bis zur Spitze des 3. Fingers: Länge $\mathrm{r}=25, \mathrm{l}=24 \mathrm{~cm}$; grösster Umfang des Oberarms $\mathrm{r}=11 \frac{3 / 4}{\text {, }}$

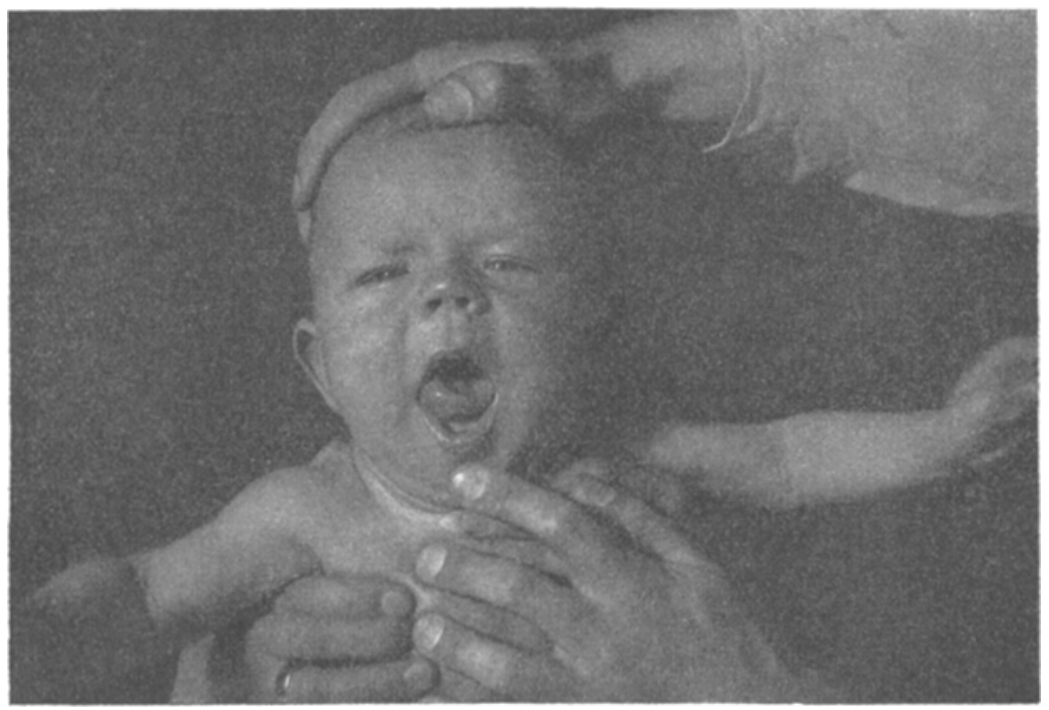

Fig. 4c.

$\mathrm{l}=11 \mathrm{~cm}$; Umfang des Unterarms $5 \mathrm{~cm}$ unterhalb der Ellenbogenspitze $\mathrm{r}=12 \mathrm{~cm}, \mathrm{l}=10,5 \mathrm{~cm}$.

Die rechte Hand ist wenig, aber deutlich grösser als die linke.

Die Vergrösserung der rechten Körperhälfte ist überall eine gleichmässige. Man sieht nirgends geschwulstähnliche Verdickungen, Knochenauswüchse oder ähnliches.

Zusammengefasst handelt es sich also um ein rechtzeitig und ohne Hindernisse geborenes, jetzt $4 \frac{1}{2}$ Monat altes Kind aus gesunder Familie, das gleich nach der Geburt eine Vergrösserung der rechten Zungenhälfte, im 3. Monat auch des Beines, im 4. Monat auch des Armes aufwies und das jetzt eine erhebliche Vergrössernng der ganzen rechten Körperhälfte zeigt. Die Extremitäten sind 
dicker und länger, der Rumpf ist deutlich asymmetrisch zugunsten der rechten Seite, Knochen und Weichteile auch der Wangengegend sind etwas mit betroffen.

Die Haut ist dabei überall unverändert, die Intelligenz erscheint intakt. Die Differenz der Körperhälften hat unter den Augen der beobachtenden Mutter bis jetzt noch zugenommen. Sichtbare Pigmentverschiebungen, Gefässerweiterungen, Schweissstörungen sind nicht nachweisbar.

Das Kind selbst bevorzugt noch keine Hand vor der anderen, die Eltern und alle Verwandten der Mutter sind jedoch ausgesprochen rechtshändig. -

Die Diagnose ist in allen drei Fällen leicht und sicher auf Hemihypertrophie zu stellen. Ernstliche Einwände gegen diese Diagnose dürften kaum angängig sein. Von den allein eventuell in Betracht kommenden Krankheiten dürfte das Lymphangiom ohne weiteres ausscheiden, da auch die Knochen von der Hypertrophie und zwar in erheblichem Maße mitbetroffen sind, da die Abgrenzung einer Geschwulst überhaupt unmöglich und die Vergrösserung unter Erhaltung der unsprünglichen Form erfolgt ist. Auch ein Trophödem im Sinne von Meige 1) - wenn wir ein solches Krankheitsbild überhaupt als selbständiges anerkennen wollen - liegt in keinem unserer Fälle vor, da die Mitbeteiligung der Knochen im Widerspruch zu der von Meige gegebenen Umgrenzung steht. Ebensowenig glaube ich, dass wir die vorliegenden Fälle in die Akromegalie einreihen können; denn wenn auch in dem ersten Falle vielleicht eine Verlängerung des 4. Fingers der linken Hand gegenüber der rechten besteht, so kann im Ganzen von einer akralen Vergrösserung keinesfalls die Rede sein, da ja die Beschränkung des abnormen Wuchses auf eine Hälfte des Körpers bzw. des Trigeminusgebiets ja gerade das Charakteristische der Krankheit ausmacht.

In symptomatologischer Beziehung weisen unsere Fälle eine Reihe von bemerkenswerten Ähnlichkeiten auf, die in früheren Fällen nur zum Teil in gleicher Weise beobachtet wurden. Auf die Einzelheiten dieser symptomatologischen Ähnlichkeiten und Verschiedenheiten einzugehen, dürfte sich erübrigen. Erwähnt sei jedoch, dass unter den nicht so wenigen Fällen von partiellem Riesenwuchs, die wir in der Literatur finden, die Begrenzung auf den halben Körper, sowie auf das halbe Gesicht wie in unseren Fällen relativ häufig beschrieben worden ist. Soweit solche Fälle einer totalen halbseitigen Hypertrophie als eine innerhalb des Lebens erworbene

1) Meige, Nouvelle Iconographie de la Salpêtrière 1899. p. 453. 
Erscheinung anzusehen sind, sind sie neuerdings von Piazza ${ }^{1}$ ) zugleich mit den vorliegenden Sektionsberichten zusammengestellt worden. Die angeboren halbseitigen Hypertrophien sind, da sie etwas häufiger vorkommen und ihre Übergänge zu den partiellen, mehr oder minder umfangreichen Hypertrophien ganzer Extremitäten oder Gliedabschnitte fliessende sind, einer zusammenfassenden monographischen Bearbeitung $\mathrm{m}$. W. in letzter Zeit nicht unterzogen worden. Sie finden sich aus der älteren Literatur bei $H$. Fischer ${ }^{2}$ ) und bei Lew in in der schon zitierten Arbeit in den Charité-Annalen zusammengestellt, im übrigen sind einige in der Literaturzusammenstellung von Ziehen ${ }^{3}$ ) mit enthalten und auch von Cagiati ${ }^{4}$ ) angeführt.

Die Beschränkung der halbseitigen Hypertrophie auf das Gesicht hat das Interesse der Forscher schon immer in hohem Maßje auf sich gezogen, besonders durch die Beziehungen zum Trigeminus und zur Hemiatrophia faciei. Zusammenstellungen solcher Fälle finden sich ausser bei Lewin, besonders bei Sabrazès und Cabanes ${ }^{5}$ ), bei Mackay ${ }^{6}$ ) und dann wieder ausführlich bei Ziehen.

Am meisten den unsrigen ähnlich sind von allen diesen diejenigen Fälle, in denen die Hypertrophie sich ausschliesslich auf eine Hälfte des Gesichts beschränkt, aber Knochen und Weichteile in gleichem Maße ergriffen hat. Es sind das die Fälle von Friedreich (1863), Heumann(1863), Passauer(1866), Fischer (1879), Bramwell(1881), Ziehl (1883), Schieck (1883), Kiwull (1890), Montgomery (1893), Dana (1893), Heath (1894), Greig (3 Fälle 1898), Hutchinson (1901), Thompson (1901), Ziehen (1909). Ein geringes Übergreifen auf solche Teile des Kopfes, die nicht rom Trigeminus innerviert werden, ist dabei oft, ein erhebliches nur selten notiert worden, ebenso wenig ein Übergreifen auf die andere Seite des Gesichts, eine Erfahrung, die mit unseren Fällen in rollstem Einklang steht. Am meisten ergriffen war von allen Teilen des Gesichts dabei fast stets die Gegend des Jochbogens und des Oberkiefers beim Knochen, der Wangen-

1) Piazza, Ein Fall von erworbener totaler rechtsseitiger Hypertrophie des Körpers. Monatsschrift f. Psych. und Neurologie. XXV. 1909. S. 497. Bd. 12.

2) H. Fischer, Der Riesenwuchs. Dentsche Zeitschr. f. Chirurgie 1880.

3) Ziehen, Über einen Fall von Hemibypertrophia faciei mit angeborenem Schwachsinn. Zeitschrift für die Erforschung des jugendlichen Schwachsinns. III. 3. 1909 .

4) Cagiati, Contributo allo studio della ipertrofia congenita. Policlinico XI. Roma 1904 .

5) Sabrazès et Cabannes, Hémihypertrophie faciale. Nouvelle Iconographie. XI. 1898 . p. 343.

6) Mackay, On so-called facial hemihypertrophy. Brain XXVII. p. 388. 
und Oberlippengegend bei den Weichteilen. Man sieht das am schönsten bei den Abbildungen von Greig $\left.{ }^{1}\right)$ Nr. 1 und 3, Sabrazès und Cabannes, Mackay, Ziehen; in ähnlicher Weise findet man aber auch die Bevorzugung dieser Gegenden bei manchen Fällen von allgemeiner Hemihypertrophie mit besonderer Beteiligung des Gesichts, z. B. bei Cagiati. Als allgemein giltige Erscheinung erwähnt diese Bevorzugung auch Mackay, der sie in 17 von 29 Fällen der Literatur feststellen konnte.

Eine so ausgeprägte Entwicklung von Exostosen, wie in unseren beiden, besonders dem 2. Falle, habe ich in der Literatur nur noch bei Mackay erwähnt gefunden. Sie wird bei uns besonders deutlich durch das vorzüglich gelungene Röntgenbild des zweiten Knaben (s. Abbildung 3c).

Etwas abweichend von der gewöhnlichen Symptomatologie ist bei unseren Fällen die Zahnbildung. Während in der Mehrzahl der Fälle, in denen überhaupt über Abweichungen der Grösse und Zahl der Zähne in dem hypertrophierten Kiefer berichtet wird, der Erfahrung gemäss ihre Zahl oder Grösse die der anderen Seite übertrifft und meist anch von einem verfrühten Durchbruch berichtet wird (Friedreich, Passauer, Bramwell, Sabrazès, Hertoghe, Finlayson) ist bei uns im ersten Fall die Zahl, im zweiten Fall ibre Grösse verringert. Vereinzelt sind derartige Erfahrungen schon früher berichtet; so fand Schieck eine Verringerung der Zahl der Zähne trotz erheblicher Grösse des einzelnen Zahnes und Heath eine Hemmung in dem Durchbruch der bleibenden Zähne. Die von Sabrazès ${ }^{2}$ ) geäusserte Annahme, dass die verfrühte Entwicklung der Zähne für eine angeborene Störung, die nicht verfrühte oder gar verspätete Entwicklung dagegen für eine erst im extrauterinen Leben erworbene Störung sprechen, ist schon von Mackay zurückgewiesen worden und findet auch in unseren Beobachtungen keine Stütze, da es sich bei uns zweifellos um angeborene Krankheitszustände handelt, trotzdem aber die Zahnentwicklung hinter der normalen Seite zurückgeblieben ist.

Extrem hochgradig ist in unseren beiden ersten Fällen ferner die Verbreiterung des Alveolarfortsatzes am Oberkiefer; sie übertrifft darin noch die Abbildungen bei Mackay und Sabrazès um ein sehr Bedeutendes. Die Herstellung von Gipsabdrücken war bei uns leider aus äusseren Gründen nicht möglich. In unserem dritten

1) Greig, Unilateral Hypertrophy. Edinburgh Hospital Reports 1898. 212. Siehe dabei auch Literaturzusammenstellung über die verschiedensten Arteu von halbseitigem Riesenwuchs überhaupt.

2) S. 366 l. c. 
Falle liegt, wie gleichfalls in der Mehrzahl der halbseitigen Hypertrophien des ganzen Körpers, eine Verbreiterung des Alveolarfortsatzes nicht vor.

Bemerkenswert ist weiter, dass in unseren Fällen 1 und 2 das erste, schon gleich nach der Geburt bemerkte Symptom der Vergrösserung eines Auges in ähnlicher Weise auch schon früher beobachtet worden ist und zwar von Ziehl, Hermanides, Cagiati und Ziehen. Die wiederholt beobachtete Protrusio eines Bulbus durch Exostosen oder andere raumbeengende Erscheinungen im hinteren Teile der Augenhöhle (Mackay, Hutchinson) darf damit nicht konfundiert werden. Wir müssen vielmehr annehmen, dass es sich in unseren und den genannten Fällen um die Entwicklung eines Hydrophthalmus gehandelt hat, der in unseren Fällen zur Enucleation, in Ziehens Fall zu nachträglicher Atrophie geführt hat. Dass diese Annahme richtig ist, wird vor allem durch den genannten Ziehenschen Fall wahrscheinlich gemacht, in dem seitens eines nambaften Ophthalmologen aus dem Befund heraus die Diagnose auf überstandenen Hydrophthalmus gestellt wurde. Diese frühe Beteiligung des Auges dürfte für den ganzen Krankheitsprozess bis zu gewissem Grade charakteristisch sein. Es ist jedoch nicht zu vergessen, dass ein angeborener Hydrophthalmus auch ohne sonstige halbseitige Hypertrophie vorkonint, wie die Zusammenstellung von MichelsohnRabinowitsch 1 ) zeigt.

Der Haarwuchs zeigte in unserem ersten Falle insofern ein eigenartiges Verhalten, als auf der kranken Gesichtsseite bei dem 12 jährigen Knaben sowohl an der Wange wie auch an der Oberlippe deutliche und ziemlich zahlreiche Barthaare sich fanden im Gegensatz zu der völlig bartfreien anderen Hälfte. Die Grenze ist dabfi an der Oberlippe ganz scharf. Die Kopfhaare waren beide ilale nicht nachweislich verändert. Dieser Befund entspricht den früheren Beobachtungen nur zum Teil, denn es wurden in einer Reibe von Fällen (Friedreich, Heumann, Ziehl, Schieck, Finlayson, Lewin, Kiwull, Sabrazès) eine grössere Starre und Steifigkeit der Kopfhaare auf der hypertrophischen Seite, in anderen Fällen keinerlei Veränderung gefunden. Dass ein zeitliches Voraufeilen des Bartwuchses, soweit ich habe feststellen können, noch niemals vermerkt ist, dürfte seinen Grund darin haben, dass diese Erscheinung eben bei einem am Beginn der Pubertätsentwicklung stehenden Knaben am besten sichtbar wird.

1) Michelsohn-Rabinowitsch, Beitrag zur Kenntnis des Hydrophthalmus congenitus. Archiv f. Augenheilkunde. Bd. 55. 1906. 
Die Pigmentflecken der Haut, die unsere beiden ersten Fälle aufweisen, sind fast ausnahmslos in allen bisher mitgeteilten Fällen in ähnlicher Weise gefunden worden, ja sie stellen das Minimum der Erscheinungen dar, da in einer recht beträchtlichen Zahl von Fällen sogar statt oder neben diesen grössere Teleangiectasien gefunden worden sind, die man sogar für die Ursache umschriebener Makrosomien angesehen hat. Dass oberflächliche Gefässerweiterungen und um andere Formen hat es sich fast nie gehandelt - niemals die Hypertrophie eines Gliedabschnittes oder einer Gesichtshälfte erklären können, dürfte eine weitere Begründung nicht nötig machen; dass aber diese Gefässerweiterungen oder Pigmentierungen eine der allgemeinen oder partiellen Hemihypertrophie koordinierte und für sie charakteristische Erscheinung sind, dürfte auch durch unsere Fälle von neuem bewiesen werden. Weitere und erhebliche Missbildungen oder Abweichungen in der ersten Anlage, wie sie sonst einige Male beschrieben sind, finden sich bei uns nicht.

Einer Erwähnung wert dürfte es weiterhin sein, dass unser zweiter Fall gerade auf der Seite der Erkrankung vor einigen Jahren eine Mittelohreiterung überstanden hat; denn wenn auch ein Zusammenhang dieser Eiterung mit der halbseitigen Gesichtshypertrophie nicht ohne weiteres erkennbar ist, so ist die gleiche Erscheinung doch so bäufig schon beobachtet worden (Friedreich, Heumann, Finlayson, Sabrazès und Cabannes, Mackay), dass es kaum möglich ist, sie für ein ganz zufälliges Zusammentreffen zu erklären.

Die Herabsetzung der Speichel- und Schweisssekretion und die vielleicht vorliegende Abschwächung der Berührungs- und Schmerzempfindlichkeit in der kranken Gesichtshälfte bei unserem ersten Patienten steht im Gegensatz zu den meisten Beobachtungen (Lewin, Kiwull, Sabrazès u. a.), die bisher mitgeteilt worden sind. Da jedoch, soweit ich übersehen kann, sonst niemals mit schweisstreibenden Mitteln und elektrischer Reizung der Speicheldrüsen Untersuchungen gemacht worden sind, so dürfte die allgemeine Beantwortung dieser Frage zurzeit noch nicht möglich sein.

Die Hypertrophie der Weichteile im engeren Sinne des Wortes entspricht in unseren Fällen ziemlich genau allen früheren Beobachtungen; sie zeichnet sich jedoch durch ihre extreme Intensität aus und dürfte kaum von früheren Fällen übertroffen worden sein. Die vorliegenden und mir zugängigen Abbildungen wenigstens bleiben sämtlich hinter unseren Fällen zurück. Dass das Fettgewebe wenigstens in Fall 1 in erheblichem Maße mitbeteiligt ist bei der Hypertrophie, das hat die Untersuchung des von den Chirurgen herausgeschnittenen Stückes der Wangenhaut gezeigt, entsprechend dem früheren Befunde 
von Kuss und Jouon (zit. nach Piazza); ob und wie weit auch das Nerven- und Muskelgewebe beteiligt sind, kann ohne Obduktion nicht erwiesen werden; das Fehlen einer wesentlichen Veränderung der elektrischen Erregbarkeit der Muskeln lässt eine nennenswerte Mitbeteiligung der Muskelfasern kaum erwarten, nach den Sektionsbefunden von Arnheim, S. Hornstein und Kuss und Jouon kann aber die Möglichkeit einer solchen Mitbeteiligung nicht in Abrede gestellt werden.

Die Mitbeteiligung der Zunge, ja ihre hochgradige und ganz früh zutage tretende Vergrösserung ist insofern bemerkenswert, als dies Symptom, wie schon Sabrazès und Cabannes erwähnen, bei den erworbenen Fällen niemals, bei den angeborenen aber recht oft beobachtet worden ist.

Der in den beiden ersten Fällen vorliegende Schwachsinn erheblichen Grades steht im Einklang mit der Beobachtung von Ziehen, der genauer auf diese Frage eingegangen ist und einem Teil der früheren Beobachter (Ziehl u. a.). Da der Schwachsinn gerade in dem, unserem Fall in seiner ganzen Symptomatologie am nächsten stehenden Fall von Ziehen auch am stärksten ausgeprägt war, so liegt der Gedanke doch nahe, dass hier nicht zufällige, sondern gesetzmässige Zusammenhänge walten.

An dem Verlauf unserer Fälle ist eigenartig, dass im Gegensatz zu der Mehrzahl der früheren Fälle, in denen eine Konstanz der Erscheinungen beobachtet wurde, der Verlauf ein dauernd progressiver war, indem alle $3 \mathrm{Mal}$ eine geringe Hypertrophie an einer Stelle - Auge, Zunge - schon bei der Geburt bestand, die Vergrösserung dieses Organs dann weiter zunahm und sich auch auf andere Körperteile ausdehnte.

Ein Behandlungsversuch ist in unseren beiden ersten Fällen seitens der chirurgischen Klinik insofern gemacht worden, als Hautteile aus Wange bzw. dem Augenlid entfernt worden sind. Der Erfolg ist jedoch kein gerade ermutigender bisher, da die so geschaffenen Narben eher eine neue Verunstaltung hervorgerufen haben, eine Beseitigung des schweren Schönheitsfehlers aber nicht gelungen ist.

Ganz besonderen Schwierigkeiten begegnet die weitere, von allen interessanteste Frage nach der Ätiologie und pathologischen Anatomie der vorliegenden Störungen, d. h. die Frage, welcher Art die Sehädigungen sind, die die hemihypertrophischen Störungen bedingen und wo wir ihren Sitz annehmen müssen.

Das Charakteristische aller Formen des partiellen Riesenwuchses dürfte unbestritten darin zu suchen sein, dass gewisse Gewebsteile, 
Organe oder Gliedabschnitte unter Erhaltung ihrer ursprünglichen Form in solcher. Weise wachsen, dass sie die homologen Teile der anderen Körperhälfte an Grösse übertreffen und so eine disharmonische Bildung des ganzen Organismus hervorrufen. Für diese krankhaften. Steigerungen der Wachstumstätigkeit müssen wir nach allgemeinen pathologischen Erfahrungen und Anschauungen als Ursache Reize annehmen, d. b. „passive Einwirkungen auf die Zellen, die diese zu einer Steigerung ihrer aktiven formativen Tätigkeit anregen." Diese alte, zuerst von Virchow in seiner Cellularpathologie ausgesprochene Auffassung vom Wesen und der Bedeutung des Reizes dürfte ebenso wie die Dreiteilung der Reize in funktionelle, nutritive und formative Reize noch heute die allgemeine Anerkennung verdienen, wie Orth zur Widerlegung der vor allem von Weigert und Ribbert gegen die Annahme formativer Reize erhobenen Einwände neuerdings ${ }^{1}$ ) in einer Beachtung verdienenden Arbeit dargelegt hat.

Dass tatsächlich durch Reize, die aus krankhaften Veränderungen entsprungen oder experimentell erzeugt sind, ein örtlich beschränktes, gesteigertes Wachstum gewisser Gewebselemente hervorgerufen werden kann, ist eine alte Erfahrung. So hat Helferich ${ }^{2}$ ) schon im Jahre 1878 auf die öfter zu beobachtende Steigerung des Längenwachstums der Röhrenknochen bei Nekrosen hingewiesen und selbst eine solche Steigerung des Längenwachstums bei Kaninchen experimentell erzeugt, indem er mit Kantharidentinktur getränkte Holzsplitterchen in die Markhöhle der Tibia versenkte. Er konnte dann ausnahmslos nachweisen, dass die so gereizte Tibia schneller wuchs als die andere nicht gereizte und zwar nicht an der Stelle der Reizung, sondern an der Epiphysengrenze. Ein völliges Analogon zu diesen Versuchen stellen auch die neueren von $\mathrm{u}_{0} \mathrm{eb}$ eingeleiteten Experimente über die sogen. künstliche Befruchtung dar, durch die erwiesen ist, dass durch chemische, ja auch durch mechanische Reizung (Mathews ${ }^{3}$ )) des unbefruchteten Eies die Entwicklung dieses Eies zu einem, wenn auch meist unvollständigen und bald absterbenden Embryo erreicht werden kann.

Dass ein abnormes, partielles Wachstum ausser durch diese mechanischen auch durch thermische Reize verursacht werden kann,

1) Joh. Orth, Rückblicke. Virchows Archiv. Bd. 200. 1910. S. 1.

2) Helferich, Über die nach Nekrose an der Diaphyse der langen Extremitätenknochen auftretenden Störungen im Längenwachstum derselben. Eine klinisch-experimentelle Studie. Deutsche Zeitschrift f. Chirurgie. X. 1878. S. 324.

3) Zitiert nach Orth. 
ist durch Penzos $\left.{ }^{1}\right)$ Versuch bewiesen, der bei einem jungen Kaninchen durch isolierte Steigerung der Erwärmung des einen Ohres während 8 Tagen ein vermehrtes Längenwachstum dieses Ohres um $13 \mathrm{~mm}$ erzeugen konnte. Nach der Freilassung des Tieres glich sich jedoch diese künstlich erzeugte Differenz der Ohrenlänge im Laufe eines Monats völlig aus. Gerade diese letztere Erfahrung beweist aber, dass der besonders von Ribbert ${ }^{2}$ ) betonte Einfluss der Zellen und Gewebe auf einander zu einem gewissen dynamischen Gleichgewicht führt, dessen dauernde Störung die Voraussetzung abgibt für die Entstehung eines persistierenden, krankbaften partiellen Wachstums.

Für die uns speziell beschäftigende Frage kommt die Einwirkung der genannten mechanischen und thermischen Reize natürlich nicht in Betracht, wohl aber der dem thermischen nahe verwandte Reiz durch Hyperämie der Gewebe. Eine solche, durch irgendwelche Ursachen bedingte Hyperämie glaubt Ribbert z. B. für die Entstehung der Geschwülste mit heranziehen zu können, indem er auf das mechanische Moment dabei und dessen das dynamische Gleichgewicht entspannende Wirkung anf die Gewebe hinweist.

In einfacherer Weise glaubte $\mathrm{F}$ ischer ${ }^{3}$ ) die verschiedenen Formen des partiellen Riesenwuchses durch eine Hyperämie erklären zu können, indem er annahm, dass durch eine schlechte Lage des Foetus im Uterus eine Störung in der venösen Zirkulation bedingt und durch die so entstandene venöse Stase in den betreffenden Organen Überernährung und gesteigertes Wachstum hervorgerufen sein könne. Er verweist zur Unterstützung dieses Gedankens auf die Tatsache, dass in der Mehrzahl der Fälle von partiellem Riesenwuchs in den vergrösserten Gliedern Teleangiektasien oder grössere Naevi vasculosi beobachtet worden sind. In dieser letzteren, für die Makrosomien an den Extremitäten zum mindesten unbestreitbaren Tatsache - ebenso wie Machenhauer ${ }^{4}$ ), Arnheim ${ }^{5}$ ), Heller ${ }^{6}$ ), S. Kalischer 7 , u. a. habe

1) Zitiert nach Cagiati, Contributo allo studio della ipotrofia congenita. Policlinico 1904. XI. Sonderabdruck S. 22.

2) Ribbert, Das pathologische Wachstum der Gewebe bei der Hypertrophie, Regeneration, Entzündung und Geschwulstbildung. Bonn 1896. S. 8. a. a. O.

3) Fischer, Der Riesenwuchs. Deutsche Zeitschrift f. Chir. XII. 1880.

4) Machenhauer, Ein Fall von angeborenem partiellen Riesenwuchs mit Berücksichtigung der Ätiologie desselben und verwandter Wachstumsabnormitäten. Zentralbl. f. innere Medizin 1896. Nr. 43. S. 1105.

5) Arnheim, Über einen Fall von kongenitaler halbseitiger Hypertrophie. Virchows Archiv. Bd. 154. 301.

6) Berliner klin. Wochenschr. 1898 :

7) Kalis cher, Über angeborene halbseitige Hypertrophie (partieller Riesen- 
ich selbst bei partiellem Riesenwuchs, und zwar an einer Zehe neulich derartige schwere Gefässveränderungen gesehen - dürfte jedoch eine Unterstiutzung seiner Anschaung, trotz ihrer Acceptierung von Sabrazès und Cabannes nicht zu sehen sein, da diese Gefässerweiterungen immer an umschriebenen Stellen der Haut liegen; ein Einfluss auf das Wachstum des ganzen Organs dürfte also kaum denkbar, die Gefässerweiterung vielmehr als eine der allgemeinen Hypertrophie koordinierte oder gar ihr untergeordnete Teilerscheinung anzusehen sein. Für die eigentliche Hemihypertrophia faciei kommt ein solcher Zusammenhang überhaupt nicht in Betracht, da, soweit ich die Literatur übersehen kann, dabei niemals krankhafte Gefässerweiterungen beobachtet worden sind und auch in unseren beiden Fällen gänzlich fehlen.

An sich dürfte jedoch der Gedanke, dass eine irgendwie entstandene Hyperämie einen Reiz darstellt, der zur Gewebshypertrophie führt, nicht a limine abzulehnen sein. Das schon erwähnte Experiment von Penzo, bei dem die durch Erwärmung erzengte Hyperämie zweifellos das wirksame Agens darstellt, beweist diese Bedeutung des vermehrten Blutzuflusses; auch erwähnt Helferich eine Beobachtung von Broca, der bei einem Aneurysma arterio-venosum am Oberschenkel nicht nur chronische Hyperämie der Weichteile, sondern auch eine Verlängerung des Femur um $2 \mathrm{~cm}$ und der Unterschenkelknochen um $1 \mathrm{~cm}$ eintreten sah.

Die Möglichkeit, auch die halbseitige Hypertrophie durch Hyperämie zu erklären, ist dabei von allen Autoren, die über diese Frage geschrieben haben, ernstlich ins Auge gefasst worden. Man hat jedoch weiter gefragt nach der Ursache einer solchen Hyperämie und sie in einer Läsion der vom Sympathicus stammenden Gefässnerven gesucht. Zuerst ist dieser Gedanke wohl von Trélat und Monod ${ }^{1}$ ) ausgesprochen w̦orden, die eine Lähmung der Vasoconstrictoren glaubten dafür verantwortlich machen zu dürfen. Unterstützt wurde dieser Gedanke wesentlich durch die Versuche von Bidder und Stirling, die bei jungen Tieren nach Sympathicus-Durchschneidung nicht nur dauernde Hyperämie, sondern auch wahre Hypertrophie des Ohres feststellen konnten.

Diesen Gedanken einer Schädigung der sympathischen Gefässnerven - sei es im Sinne einer Lähmung der Constrictoren, sei es

wuchs). Zentralbl. f. d. Grenzgebiete der Med. u. Chir. 1901. 356. Derselbe: Monatsschr. f. Psych. u. Neurol. VI. 1899. S. 432.

1) Trélat et Monod, De l'hypertrophie unilaterale partielle ou totale. Arch. générales de médecine 1869. 
einer Reizung der Dilatatoren - glaubt nun Ziehen, der in seinem Falle auch ebenso wie in unseren beiden ersten und einer Reihe früherer Fälle eine völlige Beschränkung des Prozesses auf das Gebiet des Trigeminus feststellen konnte, mit dieser Tatsache dadurch in Einklang bringen zu können, dass er eine Läsion der mit dem Trigeminus zusammen verlaufenden, ursprünglich vom Sympathicus herstammenden vasomotorischen Nervenfasern annahm. Die Schädigung selbst glaubt er zurückführen zu können auf die in seinem Falle nachweisliche abnorm lange Dauer der Geburt und die Angabe, dass die linke, erkrankte Gesichtshälfte des Kindes dabei so fest gegen das Becken gelegen haben soll, dass das Kind vom Arzte „zurückgebracht" werden musste; eine Verletzung des Ganglion Gasseri, also der Stelle, wo die Gefässnerven der Gesichtshälfte mit dem Trigeminus zuerst sich vereinigen, erscheint ihm auf diese Weise möglich und als Ursache der ganzen Krankheit zum mindesten diskutabel.

Es dürfte kaum möglich sein, gegen einen derartigen Erklärungsversuch begründete Einwände zu erheben, soweit es den fraglichen Fall selbst angeht. Diese Erklärung in gleicher Weise auch auf unsere beiden ersten Fälle auszudehnen, dürfte dagegen nicht angängig sein, da einmal ausdrücklich der schnelle und glatte Verlauf der Geburt angegeben wird und in dem anderen Falle die gleiche, wenn auch noch nicht ganz gesicherte Angabe der Stiefmutter vorliegt. Schwerer zu nehmen dürfte der Einwand sein, dass in unseren beiden Fällen schon unmittelbar nach der Geburt die Vergrösserung des Auges bemerkt wurde und bei dem weiteren Verlauf der Krankheiten eine Zurückführung dieser Vergrösserung auf eine scheinbare Vergrösserung durch Exophthalmos infolge retrobulbärer Blutung doch allzu gezwungen sein dürfte. Für unseren dritten Fall versagt schliesslich dieser Erklärungsversuch gänzlich, da es sich um eine halbseitige Hypertrophie einer ganzen Körperhälfte handelt. Dass eine Läsion des Sympathicus von wesentlicher Bedeutung ist, dagegen dürfte auch das Fehlen aller sonstigen Störungen seitens der Sympathicusfunktionen in unserem 2. und 3. Falle sprechen.

Bei dieser Sachlage dürtte m. E. für unsere und alle anderen Fälle von Beschränkung der Hypertrophie auf das Trigeminusgebiet oder eine Körperhälfte, da alle anderen Erklärungsversuche versagen unvermeidbar sein anzunehmen, dass diejenigen Reize, welche wir als Ursache des pathologischen Wachstums der Gewebe ansehen müssen, vom Nervensystem allein ihren Ursprung nehmen, und zwar ohne dass generell den Gefässnerren eine wesentliche Rolle dabei zufiele. Dass dabei irgendeine Reizung oder Läsion der peripheren Nervenfasern das Ausschlaggebende sein sollte, ist eine Möglichkeit, 
die für die auf das Gesicht beschränkten Fälle nicht ausgeschlossen werden kann. Da die gleiche Annahme für die Fälle halbseitiger Körperhypertrophie wohl als undenkbar bezeichnet werden kann, die Ähnlichkeit beider Formen der Hypertrophie aber im übrigen eine sehr weitgehende ist, so glaube ich, sind wir berechtigt, sie überbaupt als unwahrscheinlich abzulehnen, und zwar um so mehr als über die Ablehnung der Annahme besonderer trophischer Nervenfasern im Gegensatz zu den älteren Anschauungen von Samuel und Lewin zurzeit unter den Autoren Einmütigkeit herrscht.

Die weitere Möglichkeit, dass der eigentliche Ausgangspunkt der Reize im Ganglion Gasseri bzw. in den Spinalganglien zu suchen sei, ist unter den gleichen Gesichtspunkten zu beurteilen; für die auf das Gesicht beschränkte Form kann die Möglichkeit nicht abgelehnt werden; für die anderen Fälle ist sie kaum diskutabel, besonders da auch eine Entfernung der Spinalganglien durchaus nicht immer trophische Störungen hervorruft. ${ }^{1)}$

Nicht viel anders liegen die Verhältnisse bei der weiteren Möglichkeit, die Ursache der Erkrankungen in dem Endkern der sensiblen Trigeminuswurzel oder überhaupt in einer, der Syringomyelie oder $=$ bulbieähnlichen Erkrankung der Umgebung des Zentralkanals zu suchen. Bei einer solchen für die Hemiatrophie des Gesichts schon früher erwähnten Hypothese, die für die Hemihypertrophie von Mackay und in ähnlicher Weise von Greig vertreten worden ist, dürfte auch das Charakteristische aller dieser Krankheitsformen, nämlich die scharfe Begrenzung auf eine Körperhälfte und die fast gleichmässige Beteiligung der gesamten Körperhälfte in unserem dritten Fall kaum verständlich werden, abgesehen davon, dass bei der Syringomyelie m. W. hemihypertrophische Störungen niemals beobachtet sind und die Sektionsergebnisse bei den zuletzt genannten Krankheiten niemals eine Affektion dieser Gegend haben erkennen lassen.

Da also für die Hemihypertrophia corporis keine Erklärung auch nur leidlich und für die Hemihypertrophia faciei keine der genannten Erklärungen besser befriedigen kann, so glaube ich, müssen wir, trotz des energischen Widerspruchs von Lewandowsky doch die Möglichkeit ernstlich ins Auge fassen, dass die Ursache der Erkrankungen noch weiter oben, nämlich in den subcortikalen Zentren oder im Grosshirn zu suchen sei.

Der Gedanke, dass auch die nutritiven Vorgänge im weiteren

1) Siehe Lewandowsky, Die Funktionen des Nervensystems. Jena 1907. S. 108 . 
Sinne des Wortes von diesen höchsten nervösen Zentren aus beeinflusst werden, scheint mir, so fremdartig er auch zunächst anmutet, doch nicht im Widerspruch mit unseren sonstigen Erfahrungen und Kenntnissen zu stehen. Denn aus der Phylogenese wissen wir, dass die bei den einfachsten Tieren der Zelle unmittelbar innewohnende Fähigkeit, auf Beeinflussung von aussen selbsttätig mit Formveränderung zu reagieren, in der aufsteigenden Tierreihe allmählich von einem spezifischen Gewebe, nämlich dem Nervengewebe übernommen wird und dass nach der Entwicklung und Ausbildung höherer Zentren die sensiblen und motorischen Funktionen sich nicht nur weiter differenzieren, sondern dass auch die jeweilig höchsten Zentren in zunehmendem Maße Träger dieser Funktionen werden. Ich sehe kein Hindernis, warum diese Übernahme der Leistungen auf die funktionelle Tätigkeit im engeren Sinne des Wortes beschränkt bleiben und nicht auch auf die nutritiven und Wachstums.vorgänge sich ausdehnen sollte.

Es kommt hinzu, dass auch für die Ontogenese die gleiche Übernahme der Funktionen trotz der ursprünglich autochthonen, vom Nervensystem unabhängigen Entwicklung der Zellen und Organe als sicher gelten kann, und dass auch für die Wachstumsvorgänge diese Übernahme während des embryonalen Lebens verfolgt werden kann. Ich verweise dazu auf den von Cassirer ${ }^{1}$ ) zitierten Ausspruch Goldstein ${ }^{2}$ ): „Der Einfluss des Zentralnervensystems auf das Wachstum, die Frhaltung und normale Funktion der Muskulatur entwickelt sich ron einem noch nicht näher zu bestimmenden Zeitpunkt inmitten der embryonalen Periode ab in stetig zunehmendem Maße, um schliesslich im postembryonalen Leben seine ausserordentliche Bedeutung zu gewinnen." Dass dabei die höher gelegenen subcortikalen und vielleicht auch cortikalen Zentren gerade für die Ernährungsvorgänge von grosser Bedeutung sind, wird ja auch durch die klinische Erfahrung bewiesen, dass bei Querschnittserkrankungen des Rückenmarks, selbst wenn diese ziemlich hoch gelegen sind, Decubitus, also schwere Störungen der Hauternährung, kaum vermeidbar sind.

Dürften so aus allgemeinen Erfahrungen der vergleichenden Physiologie und der Pathologie keine Hindernisse einer solchen Annahme entgegenstehen, so wird sie m. E. wesentlich dadurch unterstützt,

1) Cassirer, Die trophischen Funktionen des Nervensystems. Ergebnisse der allgemeinen Pathologie und pathologischen Anatomie des Menschen und der Tiere. Herausgegeben von Lubarsch und Ostertag 1909. 13. S. 94.

2) Goldstein, Kritische und experimentelle Beiträge zur Frage nach dem Einfluss des Nervensystems auf die embryonale Entwicklung und die Regeneration. Archiv f. Entwicklungsmechanik XVIII. 1. 1904.

Deutsche Zeitschrift t. Nervenheilkunde. Bd. 44. 
dass bei der Erna S. gleichzeitig mit der Hemiatrophie des Gesichts sich sehr schwere Charakterstörungen eingestellt haben und unsere beiden Kinder mit Hemihypertrophie des Gesichts schwachsinnig sind. Die Annahme von Ziehen, dass in seinem Falle die Geburtschädigung gemeinsame Ursache des auch in seinem Falle bestehenden Schwachsinns gewesen sei, dürfte dadurch zwar nicht widerlegt werden; immerhin aber dürtte dem anderen Gedanken zum mindesten eine Wahrscheinlichkeit nicht abgesprochen werden, nämlich, dass in unseren Fällen eine allgemeine Erkrankung des Grosshirns die gemeinsame Ursache des Schwachsinns und der Hemiatrophie dar. stellt. Wenn Greig nur in 20 Proz. der früheren Fälle von Hemihypertrophie Schwachsinn vermerkt gefunden hat, so dürfte die Möglichkeit, dass auf die Untersuchung der geistigen Fähigkeiten oft nicht genügend geachtet worden ist oder die Defekte als nicht zur Sache gehörig unerwähnt geblieben sind, zum wenigsten nicht ausgeschlossen sein. Auch ist ja durchaus nicht gesagt, dass ein wesentlicher Grad von Schwachsinn mit der der Hypertrophie zugrunde liegenden Erkrankung des Grosshirns verbunden sein muss; es kann ja sehr gut in manchen Fällen die Schädigung so gering oder derartig lokalisiert sein, dass eine geistige Schwäche von nennenswerter Intensität nicht mit verursacht wird.

Auf ein letztes, m. E. sehr wesentliches Moment, das mir gleichfalls geeignet erscheint, die Annahme einer Beteiligung des Grosshirns zu stützen, nämlich die Art der lateralen Lokalisation der Hemihypertrophie in der von der funktionell wertvolleren, der Hemiatrophie in der von der funktionell weniger wertvollen Hirnhälfte innervierten Körperhälfte, komme ich nachher noch zu sprechen.

Über die Art der nach unseren Erwägungen vielleicht im Grosshirn zu suchenden Schädigungen dürften Vermutungen zurzeit noch unangebracht sein, da uns jede Unterlage für ein Urteil darüber zurzeit noch fehlt.

Die pathologische Anatomie der wenigen Fälle von balbseitiger Körperatrophie, die bisher zur Sektion gekommen sind, hat bis jetzt keinerlei Ergebnisse gezeitigt, die unsere Auffassung beeinflussen könnten. Ich verzichte daher auf die Wiedergabe dieser Sektionsergebnisse, zumal sie von Piazza an einer leicht zugänglichen Stelle neuerdings austührlich zusammengestellt worden sind.

Die bei der Zusammenstellung der Fälle von Hemiatrophie des Gesichts durch Zahlen festgelegte Tatsache einer Bevorzugung der linken Gesichtsseite gewinnt nun ein interessantes Gegenstück dadurch, dass eine gleiche Zusammenstellung der Fälle von angeborener Hemi- 
hypertrophie das Gegenteil, nämlich eine Bevorzugung der rechten Gesichts- bzw. Körperseite ergibt.

Aufgefallen und vermerkt ist diese Tatsache nach den Angaben von Fischer, der sie 1880 als die „Regel“ bestätigt, schon dem ersten Autor, der über diese Frage gearbeitet hat, nämlich Geoffroy St. Hilaire ${ }^{1}$ im Jahre 1832, in eirer mir leider nicht zugängigen Arbeit. Sie ist dann weiter auf Grund neuer Erfahrungen wiederholt bestätig worden. So fanden Lewin bei seiner Zusammenstellung von 28 Fällen das Verhältnis rechts-links $=18: 10$, Sabrazès und $\mathrm{Ca}$ bannes 11:5, Mackay 15:12 und Greig für die halbseitige Hypertrophie, die nicht auf Gesicht und Kopf beschränkt blieb, 22:16, während letzterer für die auf das Gesicht beschränkte ein solches Überwiegen nicht feststellen konnte. Auch Machenhauer und Kaliseher schliessen sich der Annahme der Bevorzugung der rechten Seite an und bezeichnen sie als das "gewöhnliche“, ohne jedoch eigene Zahlen zu bieten.

Bei einem Versuch der Nachprüfung und Ergänzung der erwähnten Zahlen durch später und selbst beobachtete Fälle zeigten sich mancherlei Schwierigkeiten. Sie lagen einmal darin, dass bei den älteren Fällen die Beschreibungen oft nicht ausreichen, um mit Sicherheit die beschriebenen Fälle als Hemihypertrophien erkennen zu lassen, im weiteren darin, dass die verschiedenen Autoren immer wieder die gleichen Fälle in ihre Statistik einrangiert haben, schliesslich und vor allem aber sind sie darin zu suchen, dass eine scharfe Begrenzung der Hemihypertrophien des Gesichts, des Körpers, einzelner Extremitäten oder Gliedabschnitte kaum durchführbar ist. Nach wiederholten vergeblichen Versuchen, eine Abgrenzung solcher umschriebenen Makrosomien von den Hemihypertrophien vorzunehmen, habe ich daher auf solche Versuche verzichtet und eine Zusammenstellung vorgenommen aller Fälle von angeborenem partiellem Riesenwuchs unter Ausschluss der erworbenen und derjenigen Fälle, in denen die Knochen allein oder die Weichteile allein betroffen waren, da hier die Möglichkeit, dass es sich um andere Prozesse, z. B. bei den Weichteilen um Lymphangiome handelte, in jedem Falle gegeben ist. Zu dem Ausschluss der letzteren hat mich besonders der von Hirschberg ${ }^{2}$ ) beschriebene Fall von halbseitiger chronischer Gesichtsschwellung veranlasst, in dem eine anatomische Untersuchung heraus-

1) Geoffroy St. Hilaire, Histoire générale des anomalies de l'organisation de l'homme. Paris 1832.

2) Hirschberg, Über das angeborene Lymphangiom der Lider, der Orbita und des Gesichts. Zentralbl. f. Augenheilkunde. XXX. 1906. S. 2. 
geschnittener Teile möglich war und ergeben hat, dass trotz der nach der Photographie sehr grossen Ähnlichkeit mit einer Hemihypertrophie doch ein Lymphangiom vorlag.

Von diesen Gesichtspunkten ausgehend, müssen wir unter den von Lewin beschriebenen Fällen als nicht geeignet die Fälle I 1, $2,3,8$ und II 2,7 ausschalten als wahrscheinlich nicht hierher gehörig. In den übrig bleibenden 23 Fällen verhielten sich die betroffenen Seiten rechts-links $=15: 8$.

Unter den weiterhin von Lewin zusammengestellten 60 Fällen von partiellem Riesenwuchs einzelner Glieder oder Gliedabschnitte sind durch Angaben der betroffenen Seiten nur 29 verwertbar. Hier beträgt das gleiche Verhältnis 17:12, im ganzen also bei allen Fällen dieses Autors $32: 20$.

Von den 29 Fällen Mackays sind 9 schon in der Statistik von Lewin mit enthalten. Bei dem Rest verhalten sich die betroffenen Seiten rechts-links $=12: 8$.

Von den 16 in Betracht kommenden Fällen von Sabrazès und Cabannes sind in den schon erwähnten Statistiken bereits 11 mit enthalten. Der Rest verbält sich rechts-links $=4: 1$.

Unter den von Greig ${ }^{1)}$ beschriebenen fremden und eigenen Fällen scheidet die Mehrzahl aus, da bei ihnen Knochen allein oder Weichteile allein betroffen sind. Bei dem Rest von 49 Fällen, die in seiner Tafel II und Tafel C zusammengestellt sind, sind nur 17 Fälle in den schon erwähnten Übersichten nicht enthalten. Dazu kommen 3 von ihm selbst beobachtete. Bei diesen insgesamt 20 Fällen ist das Verbältnis rechts-links $=11: 9$.

Ausser den von diesen 4 Autoren zusammengezählten Fällen habe ich selbst in der Literatur ${ }^{2}$ ) noch 21 gefunden, bei denen Angaben über die Rechts- oder Linkshändigkeit des Betroffenen nicht gemacht worden sind. Das Verhältnis war hierbei rechts-links $=15: 7$. Ordnen wir diese 5 Gruppen tabellarisch, dann ergibt sich: p. 212 .

1) Greig, Unilateral Hypertrophy. Edinburgh Hospital Reports 1898.

2) Die fraglichen Fälle sind folgende: Rechte Seite betroffen: Wagner (1842, Hand betroffen), Guersaut (1857, Finger), Wulff (1861, Hand), Manasse (1890, Fuss), Rie gel (1892, Körper), Hornstein (1893, Körper), Machen hauer (1896, Körper), Stembo (1896, Arm, Gesicht), Heller (1893, Körper), Arnheim (1899, Körper), Meige (1899, Bein 2 Fälle), Kalischer (1899, Extremitäten), Long (1907, Arm, Gesicht), Bouchereau (Brustdrüse). Linke. Seite betroffen: Reid (1843, Finger), Böhm (1856, Zehe), Moebiu s (1887, Körper), Duplay (1897, Bein), Vigouroux (1899, Bein), Cagiati (1904, Körper), Pagenstecher (1906, Gesicht). 
Hemihypertrophien ohne Angaben über Rechts- oder Linkshändigkeit

Lew in rechts links

Mackay

Sabrazès und Cabannes

Greig sonstige Fälle

Summa 32

20

128

4.1

119

$16 \quad 7$

Es ergibt sich also auch hier ein erhebliches, wenn auch nicht ganz so bedeutendes Überwiegen der rechten Körperhälfte, wie wir es umgekehrt bei der Hemiatrophie gefunden haben. Weitaus interessanter noch für unsere Frage scheint mir aber die folgende kleine Zusammenstellung, bei der die Rechts- oder Linkshändigkeit des erkrankten Menschen bekannt war. In der Literatur habe ich nur 3 mal Angaben über diese Frage gefunden und zwar bei Sabrazès und bei Ziehen, deren Fälle rechtshändig und Cagiati, dessen einer Fall linkshändig war. Das in dem letzteren Falle wirklich linkshändigkeit in meinem Sinne vorlag, wird durch den Zusatz des Autors erwiesen, der von dem Kinde sagt: „preferisce la prensione colla mano sinistra". Unter den von mir selbst beobachteten Fällen war in Fall 2 und 3 der Sitz der Krankheit rechts bei Rechtshändigkeit, in Fall 1 links bei Rechtshändigkeit. Ausserdem habe ich eine Makromammie, also eine einseitig abnorm grosse Mamma bei jungen Mädchen, bzw. einer nulliparen jungen Frau, im Ganzen 5 mal beobachtet. Ihr Sitz war 4 mal rechts bei Rechtshändigkeit, 1 mal links bei linkshändigkeit. Auch habe ich einen Fall von isolierter Hypertrophie der 2. Zehe links bei einem Linkshänder beobachtet. Stelle ich diese Erfahrungen zusammen, dann finden wir:

Hemihypertrophie mit Angabe über Rechts- oder Linkshändigkeit:

$$
\begin{gathered}
\text { Rechtshänder } \\
\text { rechts links }
\end{gathered}
$$

Linkshänder

\begin{tabular}{lcrr} 
Sabrazès Gesicht & 1 & - \\
Ziehen & $"$ & - & 1 \\
eig. Beob. & " & 1 & 1 \\
$" \quad$ Körper & 1 & - \\
$" \quad$ Mamma & 4 & - \\
\cline { 2 - 3 } & & &
\end{tabular}

Cagiati rechts links

eig. Beobachtung Mamma - 1 eig. Beobachtung Zehe

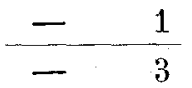




\section{III.}

Mein Interesse an der ganzen Frage der lateralen Lokalisation der Hemiatrophie und des partiellen Riesenwuchses, also derjenigen Wachstumsstörungen, die zweifellos durch nervöse Einflüsse bedingt sind, wurde ursprünglich dadurch erregt, dass ich bei meinen langjährigen Studien über Linkshändigkeit und die funktionellen Differenzen der Hirnhälften die zunächst scheinbar auf einem anderen Gebiet liegende Beobachtung machen konnte, dass die angeborenen Hemmungsmissbildungen ebenso wie die Bildung überzähliger Organe in ihrer Lokalisation durch diese Über- bzw. Unterwertigkeit der Hirnhälften mitbestimmt zu sein schienen. Ich habe daher, um diese mir zunächst sehr auffälligen Beobachtungen sicher fundieren zu können, seit 11/2 Jahren alle derartigen überzähligen Bildungen, die ich zu sehen bekam, ebenso notiert, wie die sicher als Entwicklungshemmungen anzusehenden Missbildungen und habe ausserdem in jedem einzelnen Falle nach der früher schon von mir angegebenen ${ }^{1}$ ), nachträglich erweiterten Methode die Frage geprïft, welche Hirnhälfte bei den Betroffenen als die superiore ${ }^{2}$ ) betrachtet werden muss. Das den Zählungen zugrunde liegende Material stammt zum weitaus grössten Teil aus der Nervenpoliklinik der Charité, speziell aus der mir unterstellten Kinderabteilung. Als Prototyp für die genannten Missbildungen habe ich die von mir besonders häufig beobachteten überzähligen Brustwarzen und die Syndaktylien gewählt und in jedem Falle bestimmt, auf welcher Körperhälfte diese Missbildungen ihren Sitz hatten bzw. auf welcher Körperseite die Störungen ausgeprägter waren. Ich habe dabei als rechtsseitig auch diejenigen Fälle gezählt, in denen sich rechts 2 und links eine Brustwarze oder bei der Syndaktylie rechts eine deutlich weiter nach vorn reichende Verwachsung der Zehe fand, als auf der linken Seite.

Es fanden sich bei dieser Zählung überzählige Mammillen rechts 27, links $11 \mathrm{mal}$.

Diese 27 Personen mit rechtsseitigem Sitz waren sämtlich rechtshändig, von den 11 mit linksseitigem Sitz waren 5 bestimmt rechtshändig, einer ein wenig differenzierter, jetzt aber als rechtshändig anzusehender junger Mann und 5 bestimmt linkshändig. Tch bemerke dazu, dass ich nur diejenigen Fälle gezählt habe, in denen

1) Stier, Studien über Linkshändigkeit. Monatsschrift f. Psychiatrie u. Neurologie. 1909. Bd. 25. S. 409.

2) Stier, Studien über Linkshändigkeit und die funktionellen Differenzen der Hirnhälften. Jena, Fischer 1911. S. 311 u. a. a. O. 
mir durch die Lokalisation und die Eigenart der Bildung ein Zweifel daran, dass es sich wirklich um eine überzählige Mammille und nicht etwa um einen einfachen Naevus pilosus () handelte, nicht berechtigt erschien. In der weitaus grössten Mehrzahl und allen irgendwie zweifelhaften Fällen habe ich einige von den in der Poliklinik anwesenden Kollegen um ihr Urteil gebeten und alle die Fälle ausgeschaltet, in denen von anderer Seite auch nur irgend ein Zweifel an der Natur der Bildung ausgesprochen wurde. Dass trotzdem vielleicht in einem oder dem anderen Falle die mikroskopische Untersuchung einen Naevus pilosus ergeben hätte, kann ich natürlich nicht als unmöglich ausschliessen.

Von grossem Interesse war es mir bei dieser Sachlage, als ich gesprächsweise von dem Kollegen Löhe ${ }^{2}$ ) erfuhr, dass er in der HautPoliklinik der Charité gleichfalls der Frage der lateralen Lokalisation der überzähligen Brustwarzen näher getreten war und in 22 Fällen hatte feststellen können; dass die Missbildung 21 mal ihren Sitz rechts und einmal links hatte. Diese Zählungen haben den meinigen gegenüber den Nachteil, dass die Frage der Rechts- oder Linkshändigkeit nicht geprüft wurde, aber den Vorzug, dass eine Verwechslung mit einem Naevus bestimmt ausgeschlossen ist, da Kollege Löhe in den irgendwie fraglichen Fällen eine Exzision und mikroskopische Untersuchung vorgenommen hatte.

Bei Zusammenzählung beider Zahlenreihen ergibt sich dann

$$
\text { Tabelle } 3 .
$$

überzählige Mammillen

\begin{tabular}{|c|c|c|c|}
\hline & rechts & links & \\
\hline Löhe & 21 & 1 & \\
\hline ich & 27 (alle rechtshändig) & 11 & $\begin{array}{l}\text { (5 linkshändig, } 5 \text { rechtshändig, } \\
1 \text { unsicher, da noch zu kleines } \\
\text { Kind) }\end{array}$ \\
\hline
\end{tabular}

Die einzige von mir beobachtete Hyperdaktylie, die Bildung eines doppelten Daumens, sass rechts und betraf einen Rechtshänder.

1) Die Mitteilungen von v. Bardeleben (Anatomischer Anzeiger 1892, 1893 usw.) über den Sitz der überzähligen Brustwarzen sind m. E. mit meinen Untersuchungen nicht vergleichbar, da von ihm auch solche Pigmentflecke mitgezählt sind.

2) Hern Stabsarzt Löhe sage ich für die Überlassung der Zahlen auch an dieser Stelle meinen herzlichsten Dank. 
Leider geringer als die Zahl der überzähligen Brustwarzen ist die der anderen von mir gezählten Missbildung, der Syndaktylie. Die Abgrenzung gegenüber anderen Missbildungen, ist in diesen Fällen leicht, da selbst die bis zur Verschmelzung der Knochen führenden Verwachsungen ohne Fehler als entwicklungsgeschichtlich gleichwertig mit hinzugezählt werden können und mit anderen Missbildungen eine Verwechslung kaum denkbar ist. Die Abgrenzung ist aber, besonders bei Hautverwachsungen zwischen 2. und 3. Zehe, schwierig gegenüber den physiologischen Zuständen, da schon normalerweise die verbindende Hautfalte zwischen 2. und 3. Zehe etwas weiter auf die Grundphalanx hinaufreicht als bei den übrigen Zehen, und bei der Syndaktylie alle Grade der Verwachsung von der leichtesten bis zur schwersten beobachtet werden.

Als Korrektion gegen zu grosse Subjektivität habe ich auch hier in allen der Diskussion überhaupt zugängigen Fällen mehrere anwesende Kollegen um ihre Ansicht darüber befragt, ob sie die Verwachsung schon als pathologisch ansähen, und alle diejenigen Fälle ausnahmslos fortgelassen, in denen von irgend einer Seite die Verwachsung als vielleicht noch physiologisch bezeichnet wurde. Eine weitere Schwierigkeit ergab sich dadurch, dass die Verwachsungen fast ausnahmslos doppelseitig sich finden. Da für mich aber nur die Differenzen zwischen links und rechts Interesse haben, so habe ich auch hier ungezäblt gelassen alle diejenigen Fälle, in denen die Missbildung auf beiden Seiten gleich intensiv war oder nur so geringe Unterschiede bestanden, dass mehrere Kollegen beim Betrachten zu verschiedenen Ansichten gelangten.

Der Wert der Zählungen über den Sitz der Syndaktylien ist dabei m. E. nicht so hoch zu bemessen, als bei den viel einfacheren und klareren Verhältnissen der überzähligen Brustwarzen, da eben hierbei die Differenzen zwischen rechts und links überhaupt nicht so ausgeprägt sind und die Basis der Zählungen nicht eine so objektive ist.

Die ron mir gefundenen Zahlen sind folgende:

$$
\text { Syndaktylien }
$$

rechts oder $\mathbf{r}>1 \quad 8$ (davon 5 Rechtshänder, 3 Linkshänder)

links " $1>$ r 18 (sämtlich Rechtshänder).

Ohne Unterschied gezählt sind hier die Fälle, in denen die Störungen nur an den Füssen oder gleichzeitig an Händen und Füssen. sich fanden, desgleichen die Verwachsungen zweier oder mehrerer Zehen bzw. Finger. In zweien der gezählten Fälle fanden sich ausserdem an der mehr betroffenen Hand anch Verstümmelungen der Endglieder der Finger. 
In der Literatur habe ich Zusammenstellungen über den Sitz von Schwimmhautbildungen nicht gefunden, wohl aber enthält besonders die französische Literatur eine Anzahl Beschreibungen von schwereren Hemmungsmissbildungen an den Extremitäten, die meist mit Syndaktylie verbunden sind und in der ungenügenden Entwicklung von Fingern, dem Fehlen von Fingern, Fehlen des Radius und anderem bestehen. Nach Ausschaltung derjenigen Fälle, in denen amniotische Abschnürungen an anderen Körperstellen die Genese der Störungen sicher stellte und der anderen, in denen Angaben über die von der Störung betroffene Seite fehlte oder die Störungen auf beiden Seiten gleich ausgeprägt waren, bleiben nur 6 Fälle übrig. $\left.{ }^{1}\right)$ Von ihnen war 5 mal die linke und nur 1 mal die rechte Seite in stärkerem Grade betroffen. Es ergibt sich danach im Ganzen:

Tabelle 4.

Hemmungsmissbildungen an den Extremitäten.

\begin{tabular}{lccc} 
& $r>1$ & $1>r$ \\
Literatur & 1 & 5 \\
eig. Beobachtungen & 8 (davon 3 Linkshänder) & $18 \quad$ (sämtl. rechtshändig) \\
\cline { 2 - 3 } & 23
\end{tabular}

Die linke Seite ist also $2^{1 / 2}$ mal so oft betroffen als die rechte. Zu meinem Bedauern fehlt es mir an Material, um die an sich häufigste Hemmungsmissbildung, die Hasenscharte, mit diesen Zahlen vergleichen zu können. Doch finde ich bei Weber ${ }^{2}$ ) die Mitteilung, dass Kölliker an einer von ihm leider nicht näher präzisierten Stelle auch darüber Angaben gemacht hat. Er fand unter 165 Hasenscharten 113 auf der linken Seite sitzende; das würde, wenn wir annehmen, dass die übrigen sämtlich auch einseitig und zwar auf der rechten Seite ihren Sitz gehabt haben, ein Verhältnis ergeben von rechtslinks - 52:113, das ist wieder fast genau das Verhältnis von 1:2. Die Art des Ausdruckes, dass „unter 165 sich 113 auf der linken Seite befanden" dürfte es jedoch wahrscheinlich machen, dass die übrigen nicht sämtlich rein rechtsseitig sassen und dass das Verhältnis der beiden Seiten also noch mehr zugunsten der linken Seite sich verschieben wird.

1) Die fraglichen Fälle sind beschrieben in der Iconographie de la Salpêtrière, und zwar von Allard et Lereboullet (p. 250, 1900); Souques (p. 131, 1902), Klippel et Raband (p. 239, 1903); Klippel (p. 136, 1906); Régis (p. 401, 1908); Apert et Morisetti (p. 412, 1908).

2) Ernst Weber, Ursache und Folge der Rechtshändigkeit. Halle, Marhold 1905 . S. 24 . 
Nach derselben Richtung bemerkenswert sind die von dem gleichen Autor $\left.{ }^{1}\right)$ mitgeteilten Beobachtungen ron Galippe, dass die Weisheitszähne auf der linken Seite weniger regelmässig zum Durchbruch gelangen als auf der rechten Seite und weiterhin, dass die Zähne im allgemeinen auf der linken Seite leichter zerstörenden Einflüssen anheim fallen und leichter kariös werden. Ich selbst habe bei meinem Töchterchen neulich beobachten können, dass die 3. Backzähne oben und unten rechts um einige Wochen früher durchbrachen als links. Das Kind ist ausgesprochen rechtshändig.

\section{IV.}

Stellen wir zur epikritischen Betrachtung die gewonnenen Ergebnisse zusammen, so zeigt sich zunächst, dass ganz allgemein bei den überzähligen Organen die rechte Körperhälfte, bei den Hemmungsmissbildungen die linke Körperhälfte bevorzugt ist und dass diese Berorzugung in der gleichen Weise bei den im engeren Sinnè des Wortes krankhaften hemitrophischen Störungen, der Hemiatrophie und Hemihypertrophie nachweisbar ist.

Ein klareres Bild der Verhältnisse, und die Möglichkeit einer Zurückführung dieser Tatsachen auf bekannte Erfahrungen gewinnen wir aber am besten, wenn wir die oben mitgeteilten Tatsachen noch einmal gruppieren, zur Prüfung der Frage, ob und wie häufig der rechtsoder linkseitige Sitz der Störungen mit der Superiorität oder Inferiorität der zugehörigen Hirnhälfte in Beziehung steht. Bei den nicht im engeren Sinne des Wortes krankhaften, in Abschnitt III behandelten Missbildungen müssen wir dabei alle diejenigen Fälle ausschalten, in denen ich nicht selbst die Untersuchung vorgenommen habe, da ich ausschliesslich bei diesen letzteren Fällen über diese Frage orientiert bin.

Nach Ausschaltung der von dem Kollegen Löhe beobachteten Fälle von überzähligen Mammillen (rechts-links $=21: 1$ ), der von Kölliker gemachten Mitteilungen über die Hemmungsmissbildung der Hasenscharte (rechts-links $=[52]: 113$ ) und der Literaturangaben über Syndaktylie und ähnliche Hemmungsmissbildung (rechts-links $=1: 5$ ), die lediglich das Überwiegen der rechten bei den Luxusbildungen und das Überwiegen der linken Seite bei den Hemmungsmissbildungen ergeben, zeigt sich dann folgendes Bild:

1) Ernst Weber, Ursache und Folge der Rechtshändigkeit. Halle, Marhold 1905. S. 24. 


\begin{tabular}{lcccccc} 
& \multicolumn{3}{c}{ Tabelle 5. } & \\
& Überzählige & Hyperdaktylie & Syndaktylie \\
& Brustwarzen & & & \\
& rechts & links & rechts & links & rechts & links \\
Rechtshänder & $\mathbf{2 7}$ & $\mathbf{5}$ & $\mathbf{1}$ & - & $\mathbf{5}$ & $\mathbf{1 8}$ \\
Linkshänder & - & $\mathbf{5}$ & - & - & $\mathbf{3}$ & -
\end{tabular}

Sehr viel kleiner sind die für uns verwertbaren Zahlen bei den in den Abschnitten I und II erwähnten und zusammengestellten Fällen von Hemihypertrophie, da sie mit wenigen Ausnahmen aus der Literatur genommen sind, die betreffenden Autoren aber fast niemals eine Bemerkung über die Rechts- oder Linkshändigkeit der Kranken gemacht haben. Bringe ich diese und die anderen selbst beobachteten Fälle von den früher genannten Gesamtzahlen in Abzug, dann bleibt für die Hemiatrophie ${ }^{1}$ ) ein Verhältnis ron rechts-links $=46: 94$ und für die Hemihypertrophie von rechts-links $=75: 45$. Für die übrigen Fälle aber, von denen die Rechts- oder Linkshändigkeit mir bekannt war, ergibt sich folgendes:

Tabelle 6.

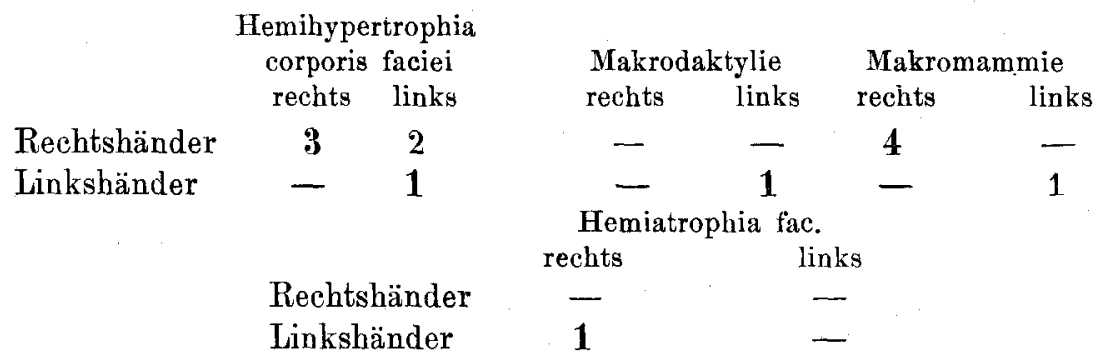

Die Gegenüberstellung der Tabellen 5 und 6 lehrt uns, dass zwischen den angeborenen Störungen, die wir als Luxusbildungen oder als Hemmungsbildungen von Organen bezeichnen müssen und den angeborenen Luxusbildungen und den im extrauterinen Leben entstandenen Atrophien von Geweben eine Übereinstimmung in der lateralen Lokalisation besteht, die noch erheblich grösser ist als es nach den Tabellen 1-4 zu sein schien. Es ist nämlich nicht nur bei allen Luxusbildungen die rechte Körperhälfte und bei den Hemmungsmissbildungen und Atrophien die linke Körperhälfte bevorzugt, sondern die betroffene Seite steht auch in be-

1) Ich habe jedoch die Beschreibungen nicht aller Fälle von Hemiatrophie in extenso daraufhin durchgelesen; es dürfte also event. noch in einem oder dem anderen Falle eine entsprechende Notiz sich finden. 
stimmten und gesetzmässigen Beziehungen zur funktionellen Differenz der Hirnhälften, als deren markantesten Ausdruck wir die Rechtsbzw. Linkshändigkeit des Menschen anzusehen gewohnt sind.

Da mich nun die schon früher erwähnten ausgedehnten Untersuchungen über die Rechts- und Linkshändigkeit gelehrt haben, dass auch die Hirnzentren für den Fuss und den Mundfacialis, ja auch für den Augenfacialis in der gleichen Weise funktionell different sind wie die Zentren für die Hand, und dass diese funktionelle Überlegenheit ebenso wie die der Sprachzentren und zum Teil der Sehzentren bei den einzelnen Menschen prinzipiell in der gleichen Hirnhälfte, nämlich beim Rechtshänder in der linken und beim linkshänder in der rechten Hirnhälfte ihren Sitz hat, so haben wir ein gutes Recht, diese im ganzen überlegene Hirnhälfte die superiore und die andere die inferiore zu nennen und die in unseren Tabellen niedergelegte Tatsache kurz so zusammenfassen, dass wir sagen:

Die Luxusbildungen und partiellen Hypertrophien haben ihren Sitz in der von der superioren Hemisphäre, die Hemmungsmissbildungen und Hemiatrophien in der von der inferioren Hirnhälfte innervierten Körperhälfte.

Diese so kurz zusammengefasste Regel gilt ohne Ausnahme für alle entsprechenden Bildungen bei den Linkshändern, wie aus den Tabellen 5 and 6 ersichtlich ist; sie gilt im Prinzip, aber nicht uneingeschränkt, bei den Rechtshändern. (Zur leichteren Übersicht sind in den Tabellen die der Regel entsprechenden Zahlen fett, die anderen mit gewöhnlicher Schrift gedruckt).

Dieser Unterschied zwischen den Links- und Rechtshändern beruht m. E. nicht auf einen Zufall. Er steht vielmehr in vollster Übereinstimmung mit einer Reihe von anderen Erfahrungen, auf die ich in meinem schon erwähnten Werke über die Linkshändigkeit ausführlich eingegangen bin. Als eine solcher Erfahrungen erwähne ich nur, dass alle Fälle einer sogen. "gekreuzten Aphasie“, wo also das Sprachzentrum in der wirklich oder scheinbar inferioren Hirnhälfte seinen Sitz hatte, bei Rechtshändern beobachtet worden sind, und ich keinen einwandsfreien. Fall gefunden habe, in dem bei einem offenbar linkshändigen Menschen das Sprachzentrum links seinen Sitz hatte. Der wichtigste Grund hierfür dürfte darin liegen, dass durch unsere Erziehung, besonders die oft nach dieser Richtung sehr intensive Erziehung in der ersten Kindheit zwar relativ oft ein "geborener" Linkshänder zum Rechtshänder̀, niemals aber ein „geborener" Rechtshänder zum Linkshänder umgew öhnt wird. Durch die von mir angegebene Untersuchungsmethode, besonders die Mitberücksichtigung der Geschicklichkeit des Fusses und des Facialis 
gelingt es nun bei einer beträchtlichen Zahl scheinbar rechtshändiger Erwachsener nachzuweisen, dass bei ihnen doch die rechte Hirnhälfte noch die superiore ist, sie also ursprïnglich als Linkshänder anzusehen waren; des weiteren aber gibt es zweifellos auch Menschen, bei denen ursprünglich ein leichtes Überwiegen der rechten Hirnhälfte besteht, die aber durch die frühe und energische Erziehung nicht nur ausgeglichen, sondern in ihr Gegenteil verwandelt wird. Die erstere von diesen Gruppen kommt besonders in Betracht bei den gekreuzten Aphasien, die zweite bei den uns hier interessierenden Fällen. Meine vielfachen Untersuchungen, besonders an kleinen Kindern, haben mir nämlich ganz besonders deutlich gezeigt, wie ausserordentlich gross die Unterschiede in der funktionellen Differenz der Hemisphären bei den einzelnen Menschen sind, dass es also, mit anderen Worten gesagt, Mensehen gibt, deren Hirnhälften ursprünglich funktionell fast gleichwertig sind und dann mit allen Übergängen auch Menschen, bei denen von Geburt an schon diese Differenzen sehr ausgeprägt und durch die Erziehung nicht beeinflussbar sind. Die Erziehung bewirkt es dann, dass im späteren Leben nur diejenigen Menschen, bei denen die rechte Hirnhälfte in beträchtlichem Maße funktionell überwertig ist, wirkliche Linkshänder bleiben, dass aber alle auch nur mit der geringsten Superiorität der linken Hirnhälfte Geborenen und ein Teil der mit geringer Superiorität der rechten Hirnhälfte Geborenen zu scheinbaren oder wirklich echten Rechtshändern d. h. Linkshirnern erzogen werden.

Bei in der frühen Kindheit erworbenen Störungen und bei anderen, von der Superiorität der Hirnhälften abhängigen Einflüssen finden wir daher Ausnahmen nur bei Rechtshändern, niemals bei Linkshändern. Für die prinzipielle Beurteilung sind daher solche dusnahmen bei Rechtshändern ohne Bedeutung, da sie nicht im Widerspruch mit der theroetischen Gesamtauffassung stehen.

Die oben aufgestellte Regel über die Lokalisation der hemitrophischen Störungen dürfte also durch ihre ausnahmslose Geltung bei Linkshändern eine Bestätigung, durch ihre nur prinzipielle, aber nicht alle Fälle umfassende Geltung bei Rechtshändern keinen Widerspruch erfahren.

Mit der Annahme der genannten Regel werden wir aber zu dem Schluss geführt, dass auch die nutritiven und Wachstumsvorgänge im Körper von der Superiorität bzw. Inferiorität der Hirnhälften abhängig sind. Diese Erfahrung aber wäre eine weitere Ergänzung des in meiner grösseren Arbeit schon geführten Nachweises, dass die Sensibilität und Schmerzempfindlichkeit, wenn überhaupt hemilaterale Differenzen bestehen, in Abbängigkeit von der funktio- 
nellen Überlegenheit der Hirnhälften auf der Seite überwiegen, die von der superioren Hirnhälfte innerviert wird. Dies zeigt sich nicht nur in einem gewissen Prozentsatz physiologischer Fälle, sondern deutlicher noch bei der gewöhnlich als hysterisch bezeichneten Hemihypbzw. Hemihyperästhesie und -algesie, die, wenn sie nicht durch örtliche körperliche Krankheiten hervorgerufen ist, in konstanter Abhängigkeit von dieser funktionellen Differemz der Hirnhälften steht. Daher das bekannte bedeutende Überwiegen der linksseitigen Hemihypästhesie.

Die Tatsache also, dass die schon in der allerersten Anlage begründete Entwicklung überzähliger Brustwarzen beim Linkshänder so gut wie immer links, beim Rechtshänder in der weitaus überwiegenden Zahl rechts ihren Sitz haben, dürfte demnach beweisen, dass schon von der Geburt an die eine Hirnhälfte die superiore ist und ron ihr stärkere Wachstumsreize ausgehen als von der anderen Hirnhälfte; die wohl auch als richtig anzunehmende, gewöhnlich frühere Entwicklung der Zähne im rechten Kiefer und der regelmässigere Durchbruch der Weisheitszähne im rechten Kiefer (Galippe) dürften als weitere Bestätigung dafür dienen, dass diese trophischen Reize auch im extrauterinen Leben weiterhin wirksam sind. Das vollkommene Spiegelbild dieser Erfahrungen wäre dann die grössere Häufigkeit der Syndaktylien, der Hasenscharten und anderen Hemmungsmissbildungen der ersten Anlage und das, wenn es sich bestätigt, frühere Cariöswerden der Zähne im linken Kiefer.

Die aus unseren Tabellen ersichtliche weitere Erfahrung, dass die angeborenen Hemihypertrophien bei Linkshändern wohl immer, bei Rechtshändern meist in derjẹngen Körperhälfte ihren Sitz haben, die von der superioren Hirnhälfte innerviert wird, würde dann unter dem gleichen Gesichtspunkt zu betrachten sein, wie die überzählige Entwicklung von Organen bei der ersten Anlage; ja auch die Hemiatrophia faciei musste trotz ihrer fast stets im extrauterinen Leben erst sichtbar werdenden Entwicklung in diese Gedankengänge mit einbezogen und als ein Manifestwerden einer ab ovo vorhandenen Minderwertigkeit der betreffenden Organe und damit der Minderleistung der betreffenden Hirnhälfte betrachtet werden.

In voller Anerkennung der Schwierigkeiten, die zunächst einer solchen Betrachtung gegenüberstehen, möchte ich doch darauf hinweisen, dass auch für die Hemiatrophie ein solcher Gedanke nicht etwas prinzipiell Neues bedeuten würde. Wir müssen vielmehr uns gegenwärtig halten, dass die Hemiatrophia faciei nicht eine Erkrankung des erwachsenen Menschen ist, sondern fast ohne Ausnahme im Kindesalter beobachtet oder wenigstens bis in das Kindesalter zurück verfolgt werden kann und meist um das 10.-12. Lebensjahr 
einsetzt. Nach einer Zusammenstellung von Beer') begann die Erkrankung unter 109 Fällen in 75,2 Proz. vor Vollendung des 2. Lebensjahrzehnts. Dass aber gerade in dieser Zeit der späteren Kindheit eine an sich minderwertige Anlage des Nervensystems manifest wird und sichtbare, in dieser Zeit scheinbar erst ausbrechende Krankheiten zeitigt, ist eine Erfahrung, die uns von den auf Heredodegeneration beruhenden Krankheiten (Dystrophien ${ }^{2}$ ), Friedreichscher Krankheit, Hérédoataxie cerebelleuse u. a.) längst bekannt und geläufig ist. Der Gedanke, dass es richtig wäre, die Hemiatrophie in diese Krankheitsgruppe hineinzunehmen, dürfte auch um so weniger auf Widerspruch stossen, als das weitere Charakteristikum der genannten Krankheiten, ihr familiäres Auftreten, auch bei der Hemiatrophie nicht gar so selten beobachtet worden ist. So hat z. B. Seeligmüller das gleichzeitige Vorkommen der Hemiatrophie bei einem Kind und ihrer Tante, und Klingmann bei einem Kind, deren Schwester, Mutter und Grossmutter beschrieben. Auch dürften die häufig und auch in unserem Falle gleichzeitig bestehenden Charakterstörungen der Annahme günstig sein, dass „die nicht harmonische Entwicklung mit teilweiser Insuffizienz" ${ }^{3}$ ) des Zentralnervensystems die Grundlage bildet für eine Krankheit, die als Ausdruck einer umschriebenen Hypofunktion gewisser an sich inferiorer Gewebselemente am ehesten uns verständlich werden kann.

Bei Annahme einer solchen prinzipiellen Bewertung würden die in der Mehrzahl der Fälle dem Ausbruch der Krankheit vorhergehenden Infektionskrankheiten oder Traumen genau so wie bei den auf Heredodegeneration beruhenden Krankheiten nicht mehr als Krankheitsursachen, sondern als auslösende Momente anzusehen sein, die an einem ron Hause aus vorhandenen Locus minoris resistentiae ansetzen und wirksam werden. Da aber jede Krankheit schliesslich die Resultante darstellt zwischen den von aussen einwirkenden Schädigungen und der inneren Widerstandskraft der Organe gegen diese Schädigungen, so dürfte es auch ersichtlich sein, dass bei der verschiedenen Intensität der schädigenden Einflüsse nicht immer, sondern nur in der überwiegenden Mehrzahl der Fälle die

1) Max Beer, Beitrag zur Kenntnis der Hemiatrophia facialis progressiva. Inaug.-Diss. Königsberg 1898.

2) Es sei daran erinnert, dass Erb ebenso wie manche Autoren vor ihm die Dystrophien ganz allgemein als Trophoneurosen bezeichnet hat, ein Name, der auch für die Hemiatrophia faciei bis hente der übliche ist.

3) So bezeichnet Jendrassik in dem „Handbuch der Neurologie" herausgegeben von Lewandowsky, II, 338 das Charakteristische der Heredodegeneration, als eine gute Verdeutschung des Wortes abiotrophy von Gowers. 
Lokalisation der Krankheit zu der allgemeinen funktionellen Wertigkeit der Hirnhälfte in festen Beziehungen steht und zwar um so eher, als diese Differenzen der funktionellen Wertigkeit beträchtliche individuelle Unterschiede aufweisen.

Für die Hemihypertrophien und angeborenen Luxusbildungen von Organen schliesst sich das Ergebnis unmittelbar an die erwähnte, aus anderen Gründen schon gezogene Schlussfolgerung, dass die Grosshirnzentren auch für die Ernährungs- und Wachstumsvorgänge in erster Linie in Betracht kommen. Die grössere Neigung zur Entwicklung von Luxusbildungen und krankhaften Hypertrophien von seiten der superioren Hirnhälfte wäre dann nur ein weiterer Ausdruck dieser allgemeinen funktionellen Überlegenheit. 